\title{
Earthquake Response of a Multi-Block Nuclear Reactor Graphite Core: Experimental Model vs Simulations
}

\begin{abstract}
Elia Voyagaki, ${ }^{\text {a* }}$ Panos Kloukinas, ${ }^{\text {a) }}$ Matt Dietz, ${ }^{\text {a) }}$ Luiza Dihoru, ${ }^{\text {a) }}$ Tony Horseman, ${ }^{\text {b) }}$ Olafur Oddbjornsson, Adam J Crewe, ${ }^{\text {c) }}$ Colin A Taylor, ${ }^{\text {d) }}$ and Alan Steer ${ }^{\text {e) }}$

The complex dynamics of a quarter-scale model of a graphite nuclear reactor core, representative of the second generation of British Advanced Gas-cooled nuclear Reactors (AGR), is investigated numerically and experimentally. AGR cores are polygonal, multi-layer, arrays of graphite bricks, with each brick allowed to rock by design relative to each other in accordance with the boundary conditions. A 35,000 degree-of-freedom (dof), non-linear finite-element model of the core created by Atkins Nuclear, was analysed on a High Performance Computing facility at the University of Bristol, and a corresponding 8 ton physical model, equipped with 3200 data acquisition channels, was built and tested on the University of Bristol 6 dof shaking table. In this paper, the two models are subjected to a series of (a) synthetic earthquake and (b) idealised harmonic input motions. The experimental data are used to compare and verify the two models and explore the dynamics of the core. A kinematic model of the response is also developed based solely on geometric constraints. The results are presented in the form of response maps and graphs. Important conclusions are drawn as to the dynamics and earthquake response of such systems, which inform numerical model validation. It is found that contrary to the case of a small number of rocking blocks which exhibit highly complex response patterns, the behaviour of the model at hand is both smooth and repeatable. An analogy between the response of the core and that of dense granular matter exhibiting particle interlocking and dilatancy is highlighted.
\end{abstract}

Kewords: nuclear core, earthquake response, finite element model, shaking table experiment, multi-body rocking, granular medium

Sponsor: EDF Energy

\section{INTRODUCTION}

The second generation Advanced Gas-cooled Reactor (AGR) nuclear power station fleet is a vital component of the UK's electricity generation mix, supplying approximately one fifth of the UK electricity supply. Now owned and operated by EDF Energy Generation, the earliest commercial AGR stations came online in 1976, with the last of the 7 stations being commissioned in 1989. Although the British Isles is a benign seismic environment, the seismic performance of UK nuclear power plants is, nevertheless, a critical design and operational factor. Understanding of the fundamental nonlinear seismic dynamics of the complex reactor systems, accounting for their actual and anticipated future states, is crucial to the modelling and decision making that underpin their continued safe operation. This paper reports findings from major comparative numerical and physical modelling study.

AGRs use graphite as a moderator and carbon dioxide as a coolant. Of particular interest in this study is the non-linear dynamics of the massive array of interlocking graphite bricks that form the reactor core structure, which houses the fuel stringers, control rods and cooling gas channels. Controlled by the boundary conditions imposed by the reactor walls, these components are free to rock without allowing overturning or excess displacements to occur. A principal seismic performance requirement is that the graphite core does not suffer post-earthquake residual distortions that would prevent insertion of the control rods required to shut down the nuclear reactions. Computational and experimental capabilities have advanced far beyond those available in the 1970s and 1980s, when the AGR fleet was conceived and designed. These advanced capabilities offer new ways to explore the graphite core's fundamental seismic performance and inform the safety assessment process. In particular, it is now possible to conduct strongly non-linear dynamic numerical analyses using models with several tens of thousands of degrees of freedom. These theoretical models can now be complemented by advanced experimental modelling that captures data from many thousands of sensors. In combination, these techniques offer new ways to validate the modelling assumptions and to explore the fundamental, complex non-linear interactions and behaviours of the multi-block graphite core.

The problem investigated in this study is the constrained rocking response of multi-body graphite column arrays connected by a shear inducing keying system in a polygonal configuration. The classical problem of rocking response involves a rigid body resting on a horizontal plane, subjected to horizontal dynamic excitation. Although apparently simple, it is a highly complex nonlinear problem that has been the subject of extensive research, both theoretical [1-15] and experimental [16-20]. The case of multi-body rocking and sliding is significantly more complex and is still not adequately understood, despite a considerable number of research efforts mostly related to earthquake stability of multidrum classical columns [21-34]. In the aforementioned studies of single- and multi-body rocking, the blocks are allowed to overturn and thus the systems investigated are inherently unstable. Stability in the classical sense of overturning is not an issue in the graphite core case, as the rocking blocks are restricted by a combination of the shear keys and the lateral

\footnotetext{
a) Senior Research Associate, University of Bristol, Queen's Building, University Walk, Bristol, BS8 1TR, UK

b) Research Fellow, University of Bristol, Queen's Building, University Walk, Bristol, BS8 1TR, UK

c) Reader, University of Bristol, Queen's Building, University Walk, Bristol, BS8 1TR, UK

d) Professor, University of Bristol, Queen's Building, University Walk, Bristol, BS8 1TR, UK

e) EDF Energy, Barnett Way, Barnwood, Gloucester, GL4 3RS, UK

* Corresponding author: e.voyagaki@bristol.ac.uk
} 
boundary of the reactor. This array of rigid blocks could, therefore, be better characterised as a special type of self-centring structural system with its dynamic response during rocking being the focus, as opposed to overturning. Of particular interest are the deformations of the core, the separations of the bricks during shaking, and the occurrence and extent of component disengagement. The latter may have safety implications during a seismic event, as severe permanent distortion of the vertical channel profiles, caused by key disengagement, could block the insertion of the control rods and prevent the safe shutdown of the reactor. It is worth mentioning that this is never the case for the intact core examined herein, as its design is inherently preventing such phenomena that might arise when component cracking is involved. However, examining the dynamics of the intact core is fundamental for understanding the physics of the problem.

Literature on the subject is scarce. Early studies were limited to one- and two-dimensional models of the High Temperature Gas-Cooled Reactor (HTGR) cores [35-36], which are simpler to treat numerically and experimentally as they involve only one major type of graphite bricks - as opposed to two in the AGR. The first method for predicting the seismic responses of AGR reactor cores was developed in the early 1980's using arrays of rigid bodies and non-linear contact elements [37]. This method was supported by an extensive set of dynamic excitation tests on small arrays of fullsize bricks and brick slices [38-39]. Seismic responses of AGR reactor cores are currently computed by GCORE [41], which uses representations of the arrays of graphite bricks similar to those used earlier [37].

The problem is both important and challenging because of its implications in the safe operation of the core, and because it goes beyond the state of the art in the area of multi-block dynamics. This publication is a first attempt to understand the fundamental mechanics of the problem in order to explain and predict the dynamic response of this complex non-linear system. Data from experimental and numerical 3D models of the reactor core, developed for this purpose, are employed. These models account for ageing effects and represent a prototype core in an advanced state of degradation late in life. ${ }^{1}$ The fundamental properties are explored numerically and confirmed through comparisons with the experimental data. Specifically, the basic behaviour and response mechanisms of the core model are explored in terms of: (a) the spatial distributions of acceleration, velocity and displacement within the core; (b) the brick-to-brick interactions; (c) the role of the keying system and the associated block interlocking; and (d) the sensitivity of response to input motion characteristics. The research reported herein helps to establish a basic level of confidence in both models (numerical and physical) and paves the way for more elaborate studies on the subject.

\section{AGR CORE PHYSICAL MODEL DESCRIPTION}

The physical model developed by the University of Bristol is analogous to the GCORE numerical model developed using the LS-DYNA ${ }^{\mathrm{TM}}$ platform [38-41]. It is built at quarter scale based on an AGR core late in life [42]. Instead of graphite, which is brittle, difficult to manufacture, dirty to handle and subject to dust production though wear during shaking, an engineering plastic called Acetal ${ }^{\mathrm{TM}}$ was selected for the physical model components as it offers increased resilience and its properties are well matched to that of graphite. A detailed presentation of the design process of the experiment is available in Dihoru et al. [43-44]. The rig is an octagonal array of bricks and keys spanning 8 layers as shown in plan and side views in Figure 1. Three types of bricks exist in the array:

- $\quad$ Lattice bricks (LB), the larger array parts, which are multi-faceted with a cylindrical bore (Figure 1c and Figure 2a). In the actual core they contain the fuel stringers.

- Interstitial bricks (IB) which are significantly smaller than the lattice and have a square profile cross section with a cylindrical bore (Figure 1c and Figure 2b). In the actual core they contain the control rods.

- $\quad$ Filler bricks which vertically separate the interstitial bricks (Figure 1b and Figure 2c).

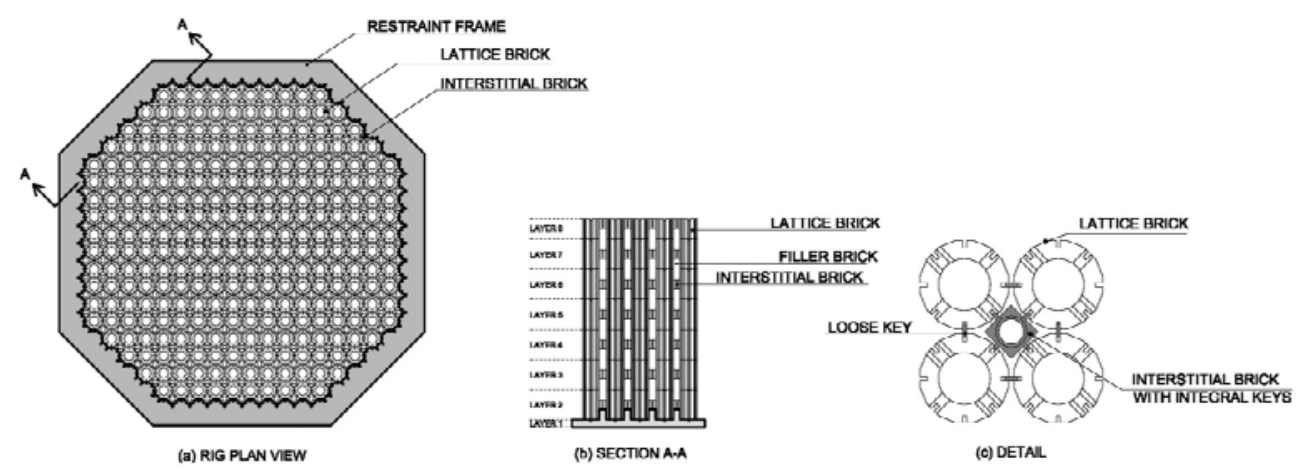

Figure 1. (a) Plan view, (b) typical cross-section and (c) detail of the multi-layer array (MLA) model of the AGR core. Base layer 1 is fixed while layers 2-8 are active. Note that the restraint frame, which is bolted to the shaking table, acts as a fixed boundary to the array to which it transmits the table motion directly.

\footnotetext{
${ }^{1}$ The theoretical and experimental approaches have only addressed arrays in their "as-designed" state, but with clearances and gaps appropriate to "late life". The "advanced state of degradation" is only relevant when investigating the effects of simulated cracks, not covered by this paper.
} 
The lattice bricks are interconnected through rectangular bearing keys (loose keys), while the connection between lattice and interstitial bricks is achieved by keys integrated with the interstitial bricks (integral keys). The loose keys are placed at the keyways along the $x$ and $y$ axes of the rig, while the integral keys are orientated $\pm 45^{\circ}$ of the $x$ axis (Figure 1c). Both key types are the same height and span across the middle third of the lattice bricks in the vertical sense (Figure 1b). The filler bricks are only vertically connected to the interstitial bricks and do not interfere with the lattice bricks. A summary of the characteristic dimensions of the brick components is provided in Table 1.

(a) LATTICE BRICK

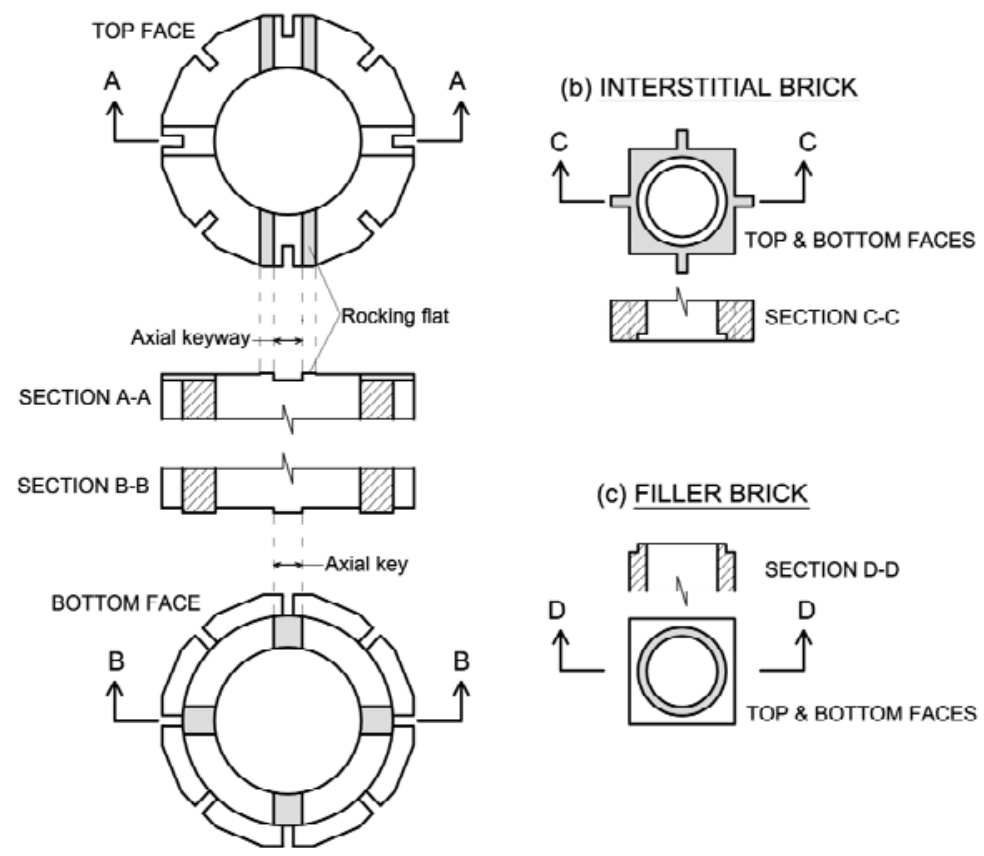

Figure 2. Brick component top and bottom faces and vertical keying systems present in the MLA rig: a) Lattice brick, b) Interstitial brick, c) Filler brick.

Table 1. Dimensions of main array components

\begin{tabular}{l|c|c}
\hline \multicolumn{1}{c}{ Component } & Dimension & $\begin{array}{c}\text { Shaking Table } \\
\text { Model (mm) }\end{array}$ \\
\hline \hline \multirow{2}{*}{ Lattice Brick } & Height & 206.30 \\
& Outer Radius & 114.75 \\
Interstitial Brick & Height & 63.54 \\
& Square Side & 47.18 \\
Filler Brick & Height & 142.75 \\
& Square Side & 47.18 \\
\hline \hline
\end{tabular}

There are 284 lattice bricks in each layer, arranged in an 18x18 octagon, totalling 2272 in 8 layers from which 1988 are loose in layers 2-8 and 284 are fixed in the base layer 1 (Figure 1b). The top layer is shorter (2/3 of the others) to level the top surface of the interstitial bricks and loose keys (Figure 1b). In each of layers 2-8 there are also 301 interstitial and 301 filler bricks. The total number of movable lattice, interstitial and filler bricks is 5901 . The total number of Acetal ${ }^{\mathrm{TM}}$ components in the rig, including loose keys, is approximately 50,000. The core model dimensions are approximately $1.4 \mathrm{~m}$ in height and $2.15 \mathrm{~m}$ across the maximum cardinal direction. The weight is approximately $60 K N$ ( $80 K N$ including the restraint system).

On the top face of the lattice bricks the two major diameters (Figure 2a) are defined by two perpendicular rectangularsection grooves (axial keyways) that allow for the bricks above and below to stand in an aligned manner. The bottom face diameters of these bricks have four rectangular protrusions called axial keys that locate in the axial keyways of an underlying brick. One of the two upper face diameters has a narrow flat ridge on each side of the keyways (shown in grey in Figure 2a), to be referred to in the ensuing as a rocking flat. The upper brick is allowed to rock along a unique rocking 
axis aligned with the rocking flat. The rocking flats are arranged in the core in four different orientations (Figure 3), so that all the lattice bricks can rock parallel to the rocking flats, that is radially towards the centre of the array. This rocking pattern in the AGR core allows the graphite array to expand and contract during heating and cooling at start up and at shut down without imposing undue stresses on the graphite blocks and steel restraints. This thermal functionality was not addressed by the shaking table experiments, which were performed at approximately constant room temperature.

The interstitial bricks, positioned between the lattice bricks, are also stacked vertically as shown in Figures $1 \mathrm{~b}$ and $2 \mathrm{~b}$. A circular recess cut at the bottom and top faces of the interstitial bricks engages with circular spigots protruding from the top and bottom faces of the filler bricks. Sliding relative to each other is thus restricted, but the interstitial bricks are allowed to rotate about their vertical axis and to rock about the edge of the circular spigot.

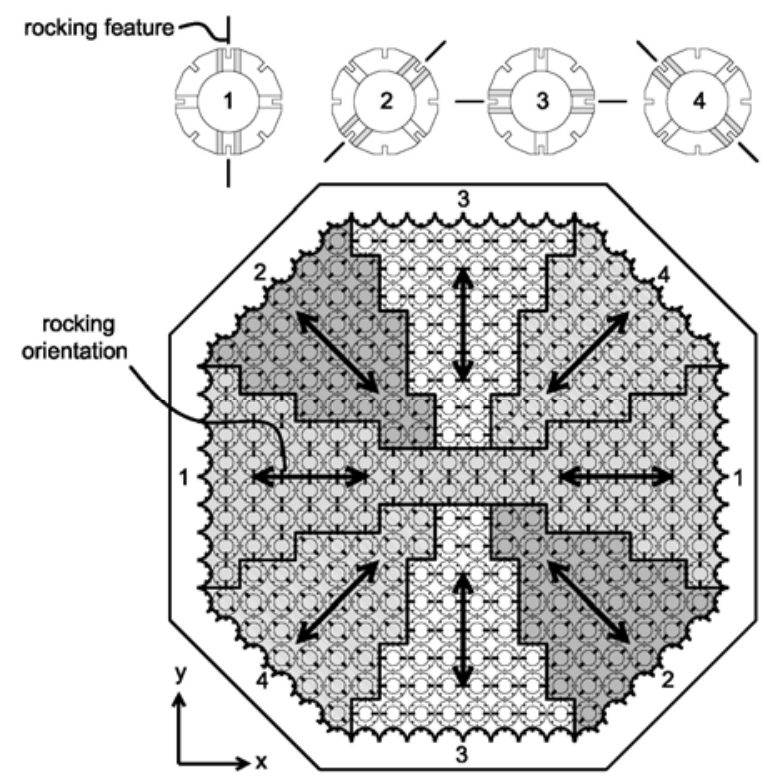

Figure 3. Brick rocking features (lines), orientations (arrows) and resulting rocking patterns in MLA array.

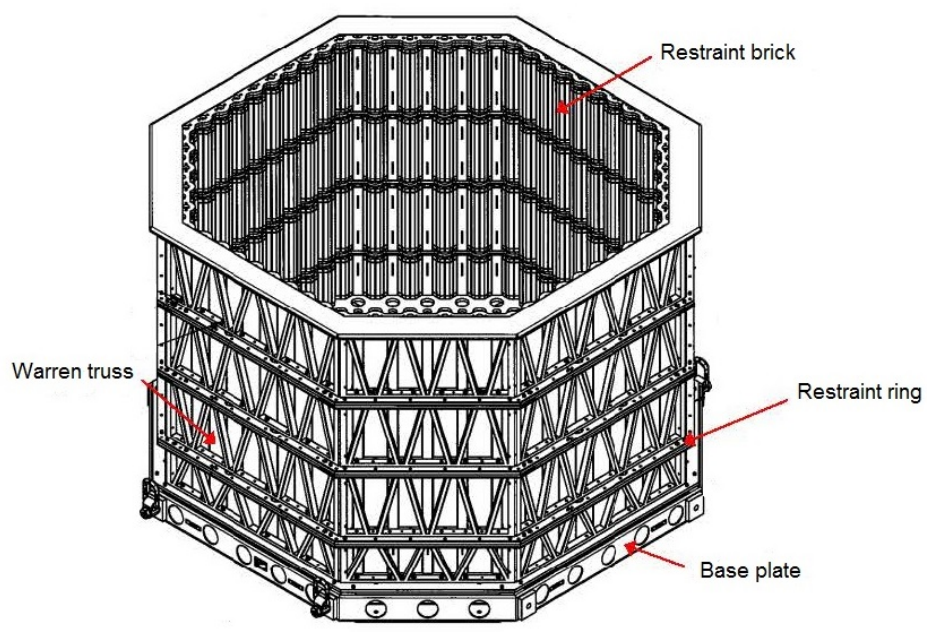

Figure 4. Restraint frame of the reactor core model.

The stability of the reactor core model is maintained by a restraint system (Figure 4). The restraint frame is an octagonal space truss whose inner wall surface is constructed of fixed half-lattice bricks and its base of a layer of fixed lattice and filler bricks allowing for the model components assembly. The restraint frame has been extensively analysed in modal tests, both numerically and experimentally (shaking table white - noise tests) to determine its structural and dynamic properties and has been shown to be seismically rigid in the frequency range of interest. The outer frame dimensions are approximately $1.7 \mathrm{~m}$ in height and $2.6 \mathrm{~m}$ in terms of major diameters. Its weight is approximately $20 \mathrm{KN}$ when empty. When testing, the restraint frame is bolted directly to the underlying platform of the University of Bristol shaking table. More information on the restraint system is available in Dihoru et al. [43-44]. 
The instrumentation involves in-house probes and measurement procedures, tailored data acquisition hardware and postprocessing software, a high-speed vision system, a network of Hall Effect transducers, accelerometers and potentiometric transducers installed in selected instrumented interstitial and lattice columns. A bespoke distributed micro data acquisition system ( $\mu \mathrm{DAQ})$ was developed by the authors, which allows the monitoring of displacements and accelerations of the instrumented components. The amount of measurement data is extensive; the monitoring of a pair of adjacent columns, one of lattice and one of interstitial/filler bricks, involves the acquisition of 250 analogue signals. The total number of channels in the rig exceeds 3200. A detailed description of the instrumentation system is available in Oddbjornsson et al. [45]. A typical schedule of experiments on a model configuration can generate over 1.5TB of digitized data.

\section{DESCRIPTION OF GCORE NUMERICAL MODEL}

An LS-DYNA ${ }^{\mathrm{TM}}$ 3-dimensional non-linear finite element model (GCORE) of the physical model was employed in the analyses. In GCORE each brick is represented as a rigid beam element along its vertical axis, with rigidly constrained rings of nodes at seven planes along its height as shown in Figure 5. The mass and inertia of each brick is assigned to a vertical beam running through the centres of the rings of nodes and has been calculated using Acetal ${ }^{\mathrm{TM}}$ properties. Characteristic values are provided in Table 2.

Contact between the bricks and through the keying system is modelled with non-linear springs and dampers which connect to the nodes of neighbouring bricks. Two types of contact are modelled in the horizontal plane through the radial keying system (Figures 5, 6 \& 7):

- Direct contact between the lattice bricks at brick vertical faces.

- Shear contact through the keying system.

Also, three contacts are modelled in the vertical plane through the axial keying system:

- Axial shear contact, which occurs at the horizontal end faces of the bricks between vertically adjacent bricks.

- Axial direct contact, which also occurs at the end faces and provides vertical support against gravitational loads to the bricks of layers 2-8.

- $\quad$ Rocking feature, which is combined with the axial direct contact and allows the rocking motion of the lattice bricks.

BRICK NODE PLANES

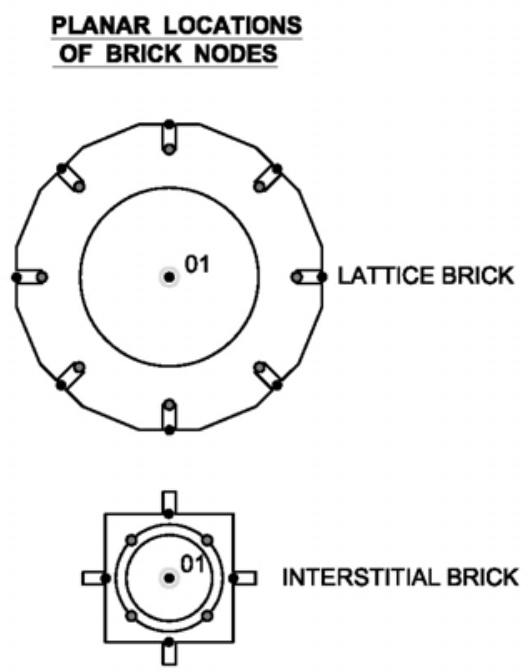

- Centre- no interactions
present in all planes, centre of mass in plane D

- Axial direct and shear contact springs and dashpots present in planes A\&G

- Radial direct springs and dashpots in planes A\&G

Radial shear springs and dashpots in planes C\&E

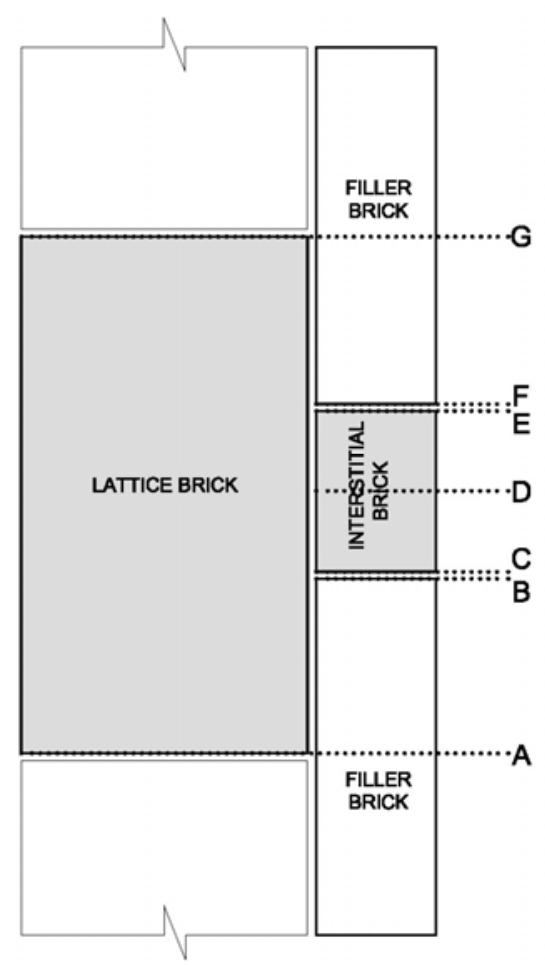

Figure 5. FE modeling of the lattice and interstitial bricks. 
Table 2. Provisional mass and inertia properties of the bricks.

\begin{tabular}{l|c|c|c|c}
\hline \hline \multicolumn{1}{c}{ Component } & $\begin{array}{c}\text { Mass } \\
{[\mathrm{kg}]}\end{array}$ & $\begin{array}{c}\boldsymbol{I}_{x x} \\
{\left[\mathrm{~kg} \cdot \mathrm{m}^{2}\right]}\end{array}$ & $\begin{array}{c}\boldsymbol{I}_{y y} \\
{\left[\mathrm{~kg} \cdot \mathrm{m}^{2}\right]}\end{array}$ & $\begin{array}{c}I_{z z} \\
{\left[\mathrm{~kg} \cdot \mathrm{m}^{2}\right]}\end{array}$ \\
\hline \hline Lattice Brick & 2.02 & $8.91 \times 10^{-3}$ & $8.91 \times 10^{-3}$ & $4.21 \times 10^{-3}$ \\
Interstitial Brick & 0.22 & $1.20 \times 10^{-4}$ & $1.20 \times 10^{-4}$ & $9.19 \times 10^{-5}$ \\
Filler Brick & 0.44 & $8.26 \times 10^{-4}$ & $8.26 \times 10^{-4}$ & $1.65 \times 10^{-4}$ \\
\hline \hline
\end{tabular}
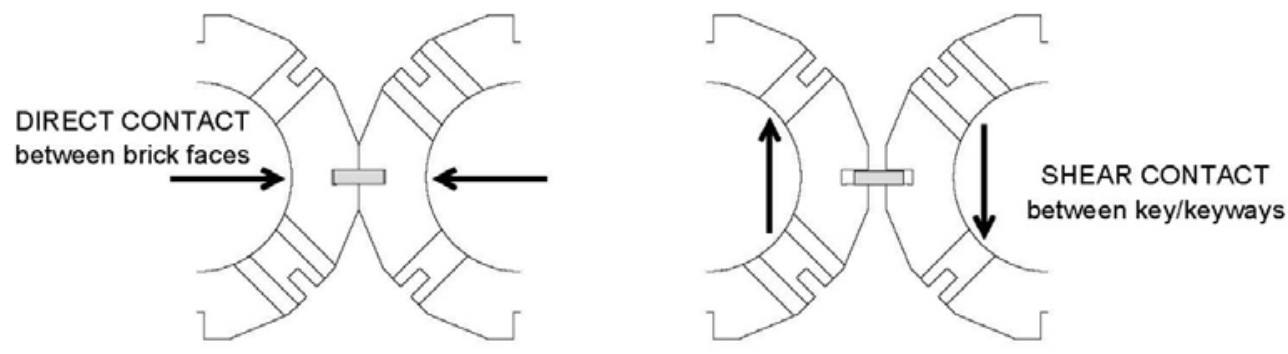

Figure 6. Types of contact between lattice bricks in the horizontal sense.

(a)

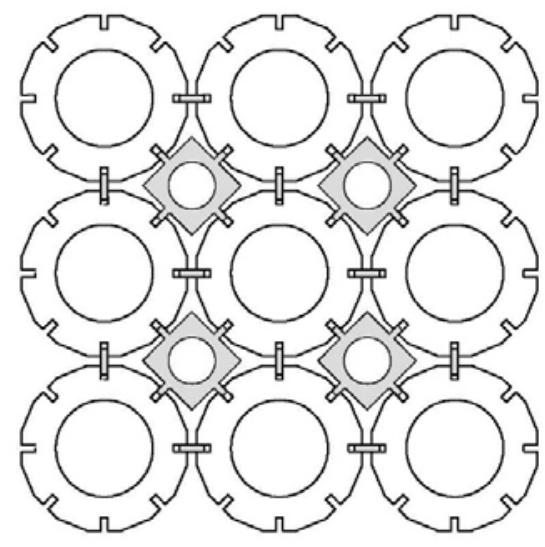

(b)

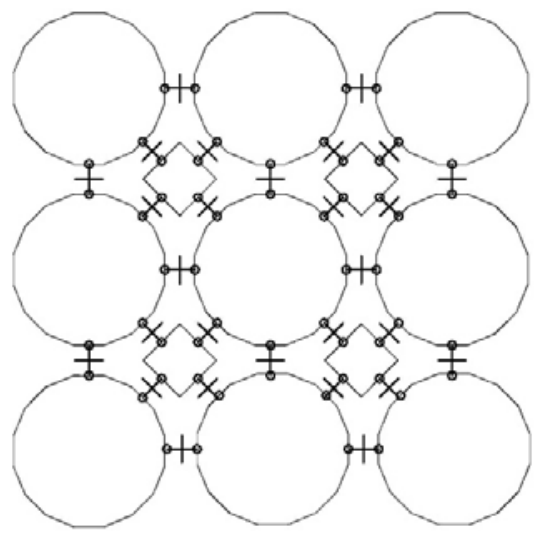

Figure 7. (a) LB-LB and IB-LB interactions through the horizontal keying system and (b) modelling by means of tensionless springs and dampers.

The modelling of the rocking feature has been simplified so that there is a 'knife edge' effect at the connection. Specifically, the rocking features are modelled by four tensionless vertical springs connecting respective nodes on the adjacent end faces of two bricks. Initially, only two diametrically opposite springs are engaged, through which the static load is transferred. The other pair of springs has its initial clearances still open; hence the springs are not engaged. This creates a statically unstable configuration and the bricks would eventually tilt until three springs are engaged. This means that the static solution has an artificial 'zig-zagging' of the bricks in a single stack, but in the dynamic case the model would represent the behaviour of the system accurately.

The impact stiffness and any gaps or clearances between bricks are represented in the non-linear force-displacement curves used to define the behaviour of the springs and dampers (see Tables 3 and 4). The damping force is assumed to be proportional to impact velocity. For direct contact the stiffness springs are only active in compression. The term "gap" refers to the direct distance between two brick faces, while the term "lost motion" refers to the distance a brick can move past another before the keying system engages. The offset apparent in Table 3 refers to the distance a key is misaligned from the center of the keyway. In the case of the intact array presented in this study, the offset values in the numerical model are zero. Note that the keys are modelled via springs in GCORE, while being physically modelled in the rig. In the physical model the location of the keys in the keyways is random and shaking results in different initial conditions for every test. The geometry and the dimensions of all rig and numerical model components, as well as their density and stiffness properties have been defined accounting for the effects of aging under fast neutron irradiation and radiolytic oxidation (e.g. increased clearances/ gaps due to material loss). Hence, both the experimental and the numerical models represent a prototype core late in life. These aging effects have been considered uniform across the graphite core. 
Table 3. Force - displacement and velocity relationships

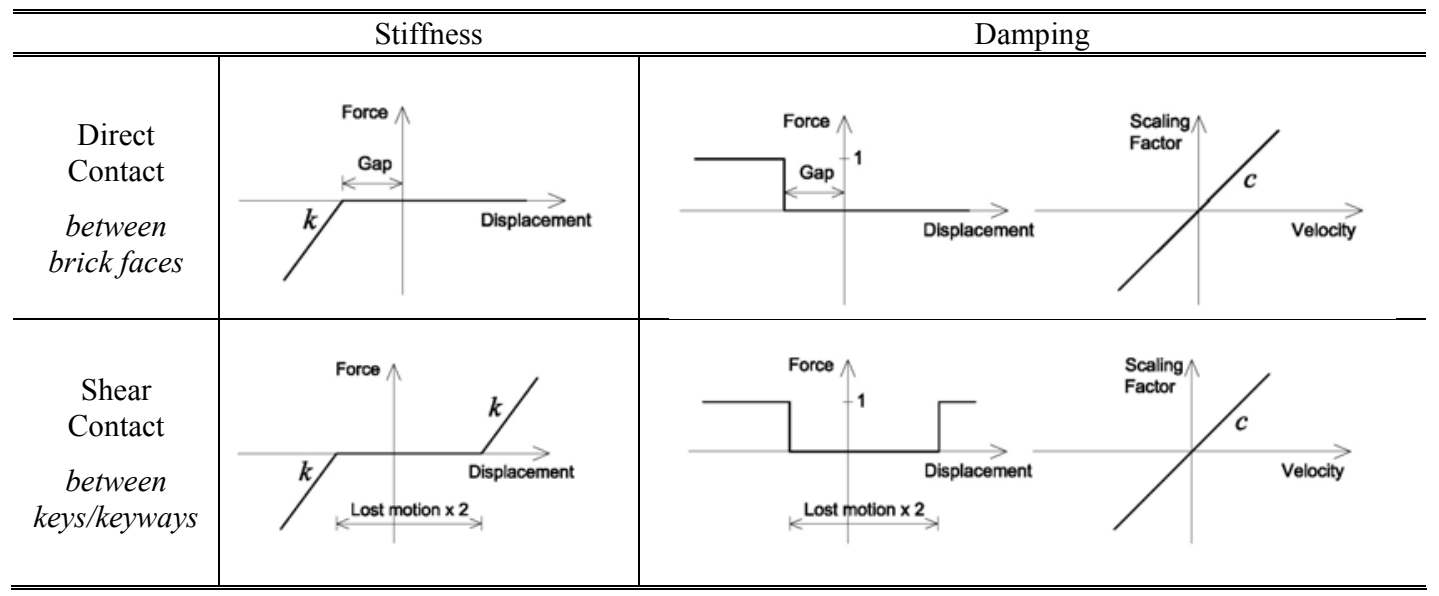

Table 4. Contact gaps and lost motion values

\begin{tabular}{cc}
\hline \hline \multicolumn{2}{c}{ Horizontal Direct Contact Gap $(\mathrm{mm})$} \\
\hline \hline LB-LB interaction & LB-IB interaction \\
3.96 & 2.89 \\
\hline \hline \multicolumn{2}{c}{ Horizontal Shear Key/Keyway Lost Motion $(\mathrm{mm})$} \\
\hline \hline LB-LB interaction & LB-IB interaction \\
0.43 & 0.14 \\
\hline \hline
\end{tabular}

Table 5. Summary of stiffness $(k)$ values considered in the GCORE numerical model.

\begin{tabular}{|c|c|c|c|}
\hline \multicolumn{4}{|c|}{ " Direct Contact Stiffness $(\mathrm{kN} / \mathrm{m})$} \\
\hline \multicolumn{2}{|c|}{ Horizontal } & \multicolumn{2}{|c|}{ Vertical } \\
\hline LB-LB spring & $7.74 \times 10^{3}$ & LB-LB spring & $7.74 \times 10^{3}$ \\
\hline LB-IB spring & $4.42 \times 10^{3}$ & & \\
\hline \multicolumn{4}{|c|}{ Shear Key/Keyway Contact Stiffness $(\mathrm{kN} / \mathrm{m})$} \\
\hline \multicolumn{2}{|c|}{ Horizontal } & \multicolumn{2}{|c|}{ Vertical } \\
\hline LB-LB spring & $2.41 \times 10^{3}$ & LB-LB spring & $2.41 \times 10^{3}$ \\
\hline LB-IB spring & $3.01 \times 10^{3}$ & & \\
\hline
\end{tabular}

Table 6. Summary of contact damping coefficient $(c)$ values considered in the numerical model.

\begin{tabular}{|c|c|c|c|}
\hline \multicolumn{4}{|c|}{ Direct Contact Damping $(k N \mathrm{~s} / \mathrm{m})$} \\
\hline \multicolumn{2}{|c|}{ Horizontal } & \multicolumn{2}{|c|}{ Vertical } \\
\hline LB-LB dashpot & 0.76 & LB-LB dashpot & 0.761 \\
\hline LB-IB dashpot & 0.21 & & \\
\hline \multicolumn{4}{|c|}{ Shear Key/Keyway Contact Stiffness $(k N$ s/m) } \\
\hline \multicolumn{2}{|c|}{ Horizontal } & Verti & \\
\hline LB-LB dashpot & 0.425 & LB-LB dashpot & 0.425 \\
\hline LB-IB dashpot & 0.172 & & \\
\hline
\end{tabular}

The direct and shear contact stiffness values were calculated to represent the compressive stiffness of two bricks, based on experimental data on impacts between two bricks of the actual prototype core and scaled for the purposes of the test. Direct contact between two lattice bricks $(L B-L B$ interaction) is not kinematically admissible in the case of an intact core, like the one studied in this paper. However, in the case of a core containing cracked components direct contact is achieved and corresponding contact springs are activated. The scaling factor for the contact stiffness is derived from the 
simplified stiffness ratio of full scale graphite to quarter scale Acetal ${ }^{\mathrm{TM}}$, calculated using the Young's modulus and the geometric scaling ratio [45]. Tables 5 and 6 provide the stiffness and damping coefficient values considered in the numerical model.

The calculated stiffness $k$ is applied to a single spring connecting two rigid bodies. Such single springs are located in two planes (A, G, Figure 5) at the top and bottom of the lattice bricks and thus the effective stiffness is doubled if full side contact occurs.

The damping coefficient, $C$, has been calculated assuming a constant value for the coefficient of restitution $\varepsilon$, approximately equal to 0.65 , using the following expression

$C=2 \zeta \sqrt{k \frac{m_{1} \cdot m_{2}}{m_{1}+m_{2}}} \quad, \quad \zeta=\frac{|\ln \varepsilon|}{\sqrt{\pi^{2}+\ln \varepsilon^{2}}}$

in which $m_{1}$ and $m_{2}$ are the masses of two adjacent bricks. Eq. (1b) is derived from free rocking considerations [20].

The main assumptions adopted in the numerical model are summarised as in: (a) the bricks are rigid; (b) deformations are sufficiently small so that geometric nonlinearity can be ignored; (c) the direction of springs and dampers does not follow the rotation of bricks. No rotational springs/dampers have been created at the keyways; (d) damping at contacts is compression-only linear viscoelastic. No additional (e.g. Rayleigh) damping is considered; (e) at the interfaces between vertically adjacent lattice bricks, only axial keys are modelled; (f) no damage occurs to the bricks or the keying system; $(\mathrm{g})$ the rocking feature on lattice bricks is modelled as a knife edge, without sliding and associated frictional effects; (h) the model restraint frame is assumed rigid; (i) the fuel stringers and control rods are not modelled; (j) the excitation is purely horizontal; In addition, given the restraining action of the boundaries which prevent large movements, small deformation analysis is adopted.

The nonlinear integration is performed by the LS-DYNA ${ }^{\mathrm{TM}}$ explicit dynamic solver using the Central Difference method. The solving time step $\Delta t$ is $0.5 \times 10^{-5} \mathrm{~s}$. To balance refinement with data-overload, the output request rate varies during the signal, usually between 0.1 to $0.01 s$ depending on the circumstances. The numerical runs were performed on the 3568 core BlueCrystal phase 3 HPC facility at the University of Bristol Advanced Computing Research Centre. Average CPU time per simulation for 5901 rigid bodies, encompassing 35,406 non-linear dynamic degrees of freedom in three dimensions, and utilising 32 processor nodes in parallel, is about 4 hours for a $20 s$ long input record. Over 100 runs were conducted as part of the herein reported analyses, corresponding to 800 hours of CPU time.

\section{INPUT MOTIONS \& ANALYSES SUMMARY}

The site-specific earthquake motion primarily considered in the analyses has a $10^{-4}$ probability of occurrence per annum and is referred to as $H P B$. The unscaled $H P B$ accelerogram and PSA spectrum are shown in Figure 8a. The input motion has peak acceleration, velocity and displacement of $A_{g}=4 \mathrm{~m} / \mathrm{s}^{2}, V_{g}=0.19 \mathrm{~m} / \mathrm{s}$ and $D_{g}=0.027 \mathrm{~m}$, respectively, and was scaled in terms of amplitude from 20 to $160 \%$ to cover a variety of intensities. In terms of frequency, the record was used both unscaled and scaled with a scaling factor of 2 to comply with the quarter-scale geometry using $N_{f}=N^{0.5}, N_{f}$ being the frequency scaling factor and $N(=4)$ the geometric scaling factor. Further discussion on the scaling procedures is available in Oddbjornsson et al. [45].
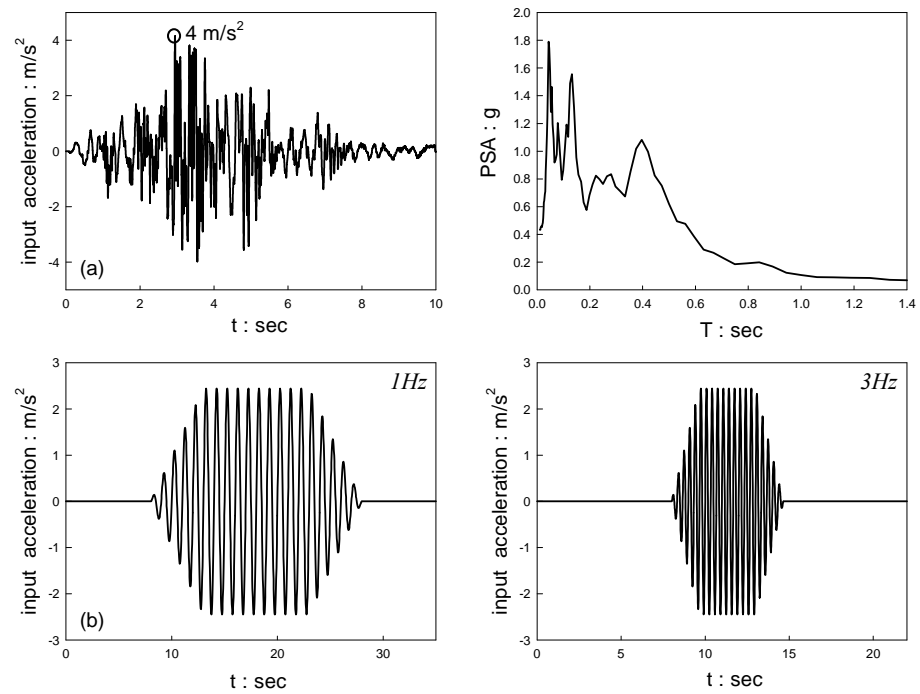

Figure 8. (a) Full-scale HPB seismic acceleration time history and response spectrum for the $10^{-4}$ seismic event and (b) examples of sinusoidal dwells used in the analyses. 
For exploring the dynamic properties of the array and for a better understanding of the mechanics of the response, a family of harmonic input motions was considered. Sinusoidal dwells with varying acceleration amplitude, ranging from $0.05 \mathrm{~g}$ to $0.4 \mathrm{~g}$ and frequency, from 0.5 to $6 \mathrm{~Hz}$, leading to fifty combinations, were employed in the numerical simulations at model scale. Examples are given in Figure 8b. The specific frequency range falls within that of the design seismic events [45]. A subset of 20 acceleration-frequency combinations within that domain was available from the experimental data and is used for comparisons.

\section{RESULTS}

A set of comparisons between predicted (numerical) and recorded (experimental) data is initially presented to validate the numerical code. Both earthquake and harmonic inputs are used as mentioned in the previous section. Following the validation section that demonstrates the fundamental agreement between the two models, the set of harmonic excitations is mainly employed to explore the physics of the problem with emphasis on numerical simulations that have the advantage of providing response data for all array locations.

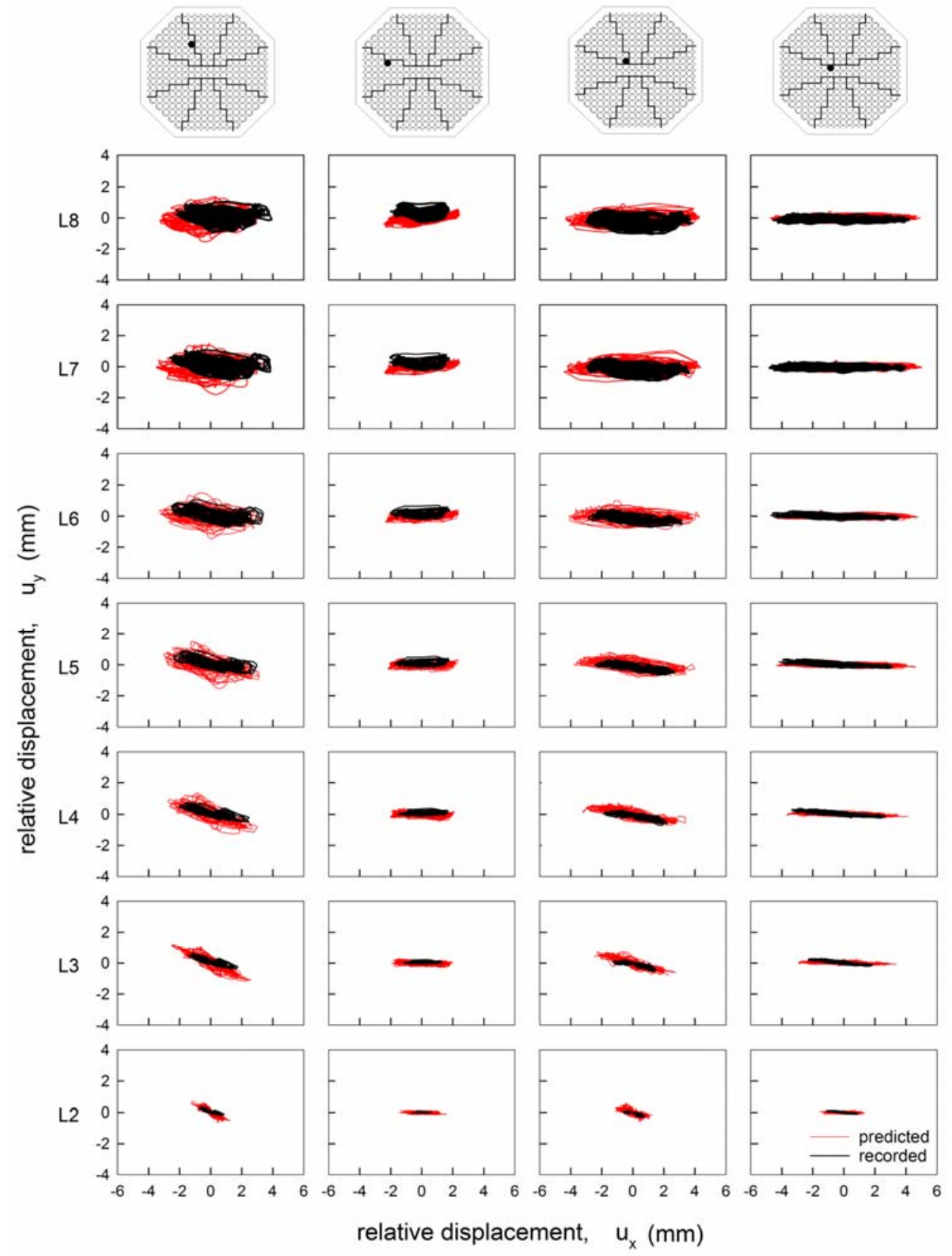

Figure 9. Displacement response paths along the height of four selected lattice brick columns (denoted by the black dots in the array plan) during the HPB input motion along $x$. Comparison of numerical results (in red) versus experimental data (in black). Layer numbers denoted L2, L3 etc. 
Figure 9 shows displacement response paths along the height of four instrumented lattice brick columns due to the HPB acceleration input motion applied in the $x$ axis. Red lines represent numerical predictions while black lines represent experimental recordings. In the second and fourth column, the rocking orientation of the bricks is parallel to the input excitation while in the first and third column it is 45 degrees. Response perpendicular to the direction of the input motion is expected due to the geometric component layout (see also rocking orientation patters in Figure 3). As illustrated in the figure, the rocking patterns dominate the response of the lattice bricks on the lower array layers. For example, in the first and third columns of the figure, which refer to stacks of bricks with 45 degree rocking patterns, the orientation of the response loops is close to 45 degrees in layers 2 and 3 and gradually aligns with the input motion orientation (which is along the $x$ direction) at the upper layers of the array. This stems from the kinematics of the array as explained in the following sections. As a result, the overall response of the columns takes the form of an $x-y$ displacement path, the area of which depends on the position of the lattice column in the array with respect to the intrinsic rocking orientation, the layer and the distance from the rigid boundary. This behaviour is well captured both by the numerical and the physical model, despite the differences observed in terms of peak displacement values and loop areas.
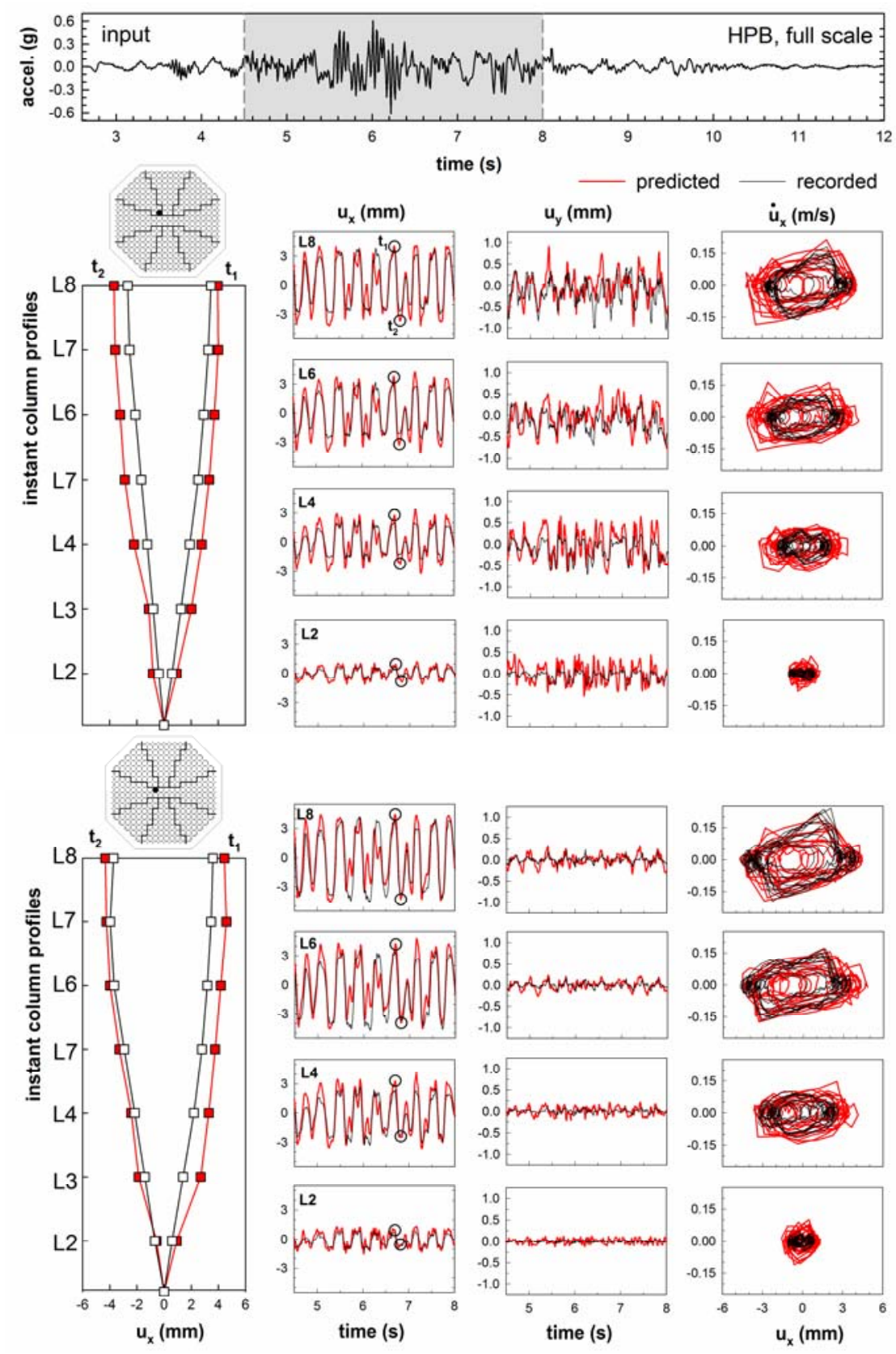

Figure 10. Comparison of numerical (red) to experimental (black) data for relative displacement time histories; and velocity - displacement space, along $x$ and $y$, for two adjacent LB columns located in the centre of the array having different rocking orientations, during the HPB input motion along $x$. 

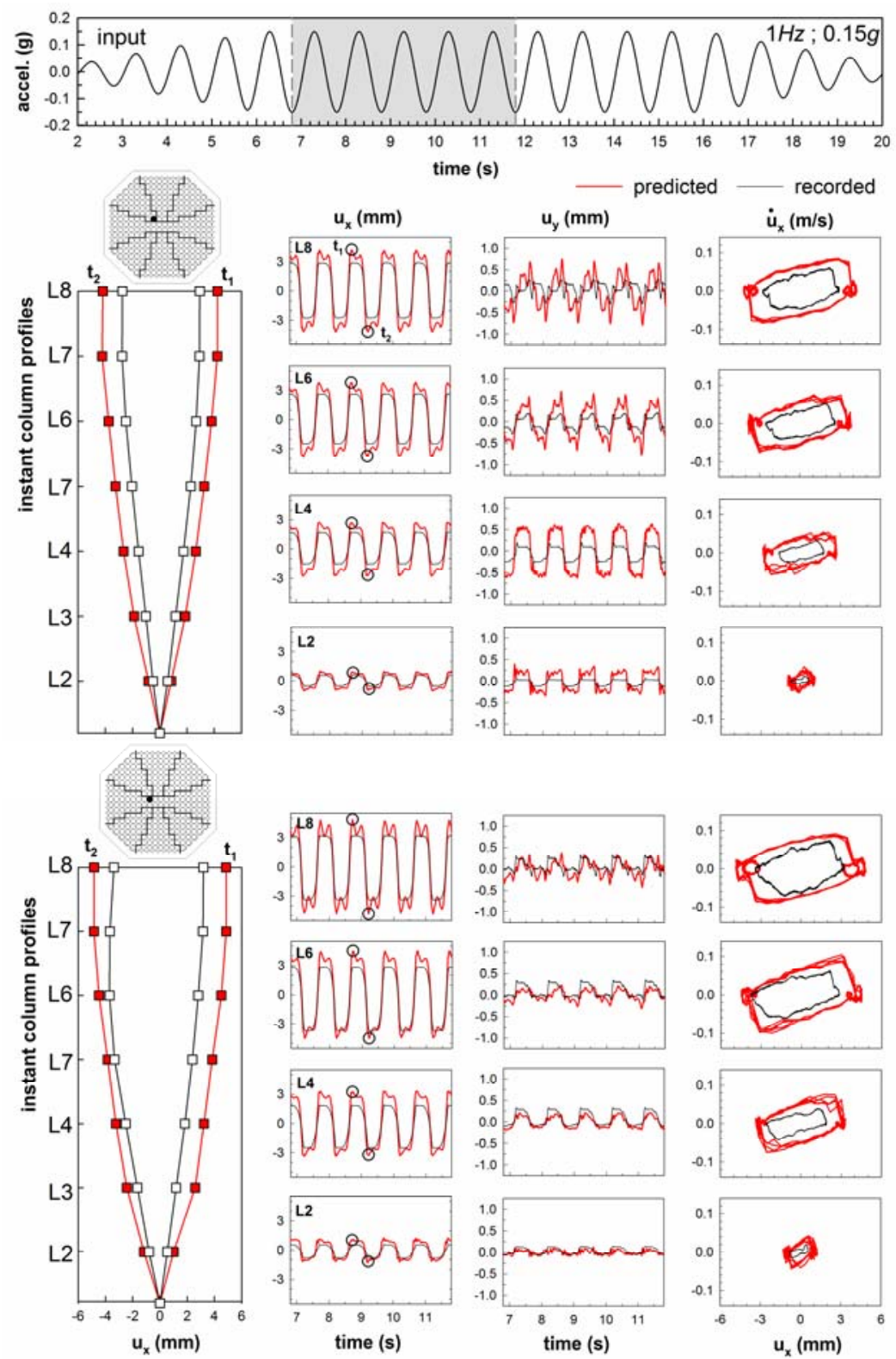

Figure 11. Comparison of numerical (red) to experimental (black) data for relative displacement time histories; and velocity - displacement space, along $x$ and $y$, for two adjacent LB columns located in the centre of the array having different rocking orientations, during a sinedwell input with $A_{g}=0.15 g, f=1 \mathrm{~Hz}$, along $x$.

Of interest are the response patterns of the last two monitored locations (third and fourth columns of diagrams) shown in Figure 9. These are adjacent, directly interacting through the radial keying system, located at the central and most mobile area of the array and at the same time have different rocking orientations. Further comparisons of the behavior of these particular bricks are given in Figures 10 and 11 for earthquake and harmonic inputs, respectively. Typical instant column profiles are illustrated, to describe the deformations of the LB stacks at the time of maximum and minimum displacement of the top layer. Lattice brick columns seem to follow a "first mode" shape parallel to the excitation. The response displacement time histories are showing good agreement between the numerical and the experimental data. Note that the out of plane $\left(u_{y}\right)$ response is significantly smaller than the one parallel to the excitation $\left(u_{x}\right)$, thus different scaling is used in the plot axes. The dependence of the response on the rocking characteristics is again evident in both predicted and measured behavior. This is demonstrated by the larger $u_{y}$ response of the column located at the 45-degree rocking section. As expected, the two columns are responding similarly parallel to the input excitation $u_{x}$, but the out of plane response is dictated by the rocking orientation. The velocity-displacement space plots also reveal a fundamental agreement between the two models. In general, although the numerical model is over-predicting the actual response, it captures the principal dynamic behaviour of the physical model, lending support to the overall numerical model validity It is noted that the 
comparisons with the experimental results can be improved by lowering the coefficient of restitution (increasing damping during impact) until discrepancies are minimised, but such a fitting procedure lies beyond the scope of this work.

Besides studying the response of separate brick columns, the complex interactions between the adjacent columns through the keying system, in compliance with the kinematic constraints dictated by geometry, are explored. A numerical investigation of these interactions is presented in Figures 12-14. Acceleration, relative velocity and relative displacement response time histories of five top layer neighbouring lattice bricks, located along the array $x$ symmetry axis, spanning from the array perimeter towards the centre, are depicted in Figure 12. The input excitation considered is a $1 \mathrm{~Hz}$ sinedwell motion with a higher acceleration amplitude of $0.25 \mathrm{~g}$ to capture the brick-to-brick impacts. Only a segment of the time histories during a characteristic steady state response cycle is plotted. The mass-normalised kinetic energy flow rate $\left(\dot{u}^{2} / 2\right.$, $t$ ), the state space $(\dot{u}, u)$ and the inertial force - displacement space $(\ddot{u}, u)$ loops have also been produced.
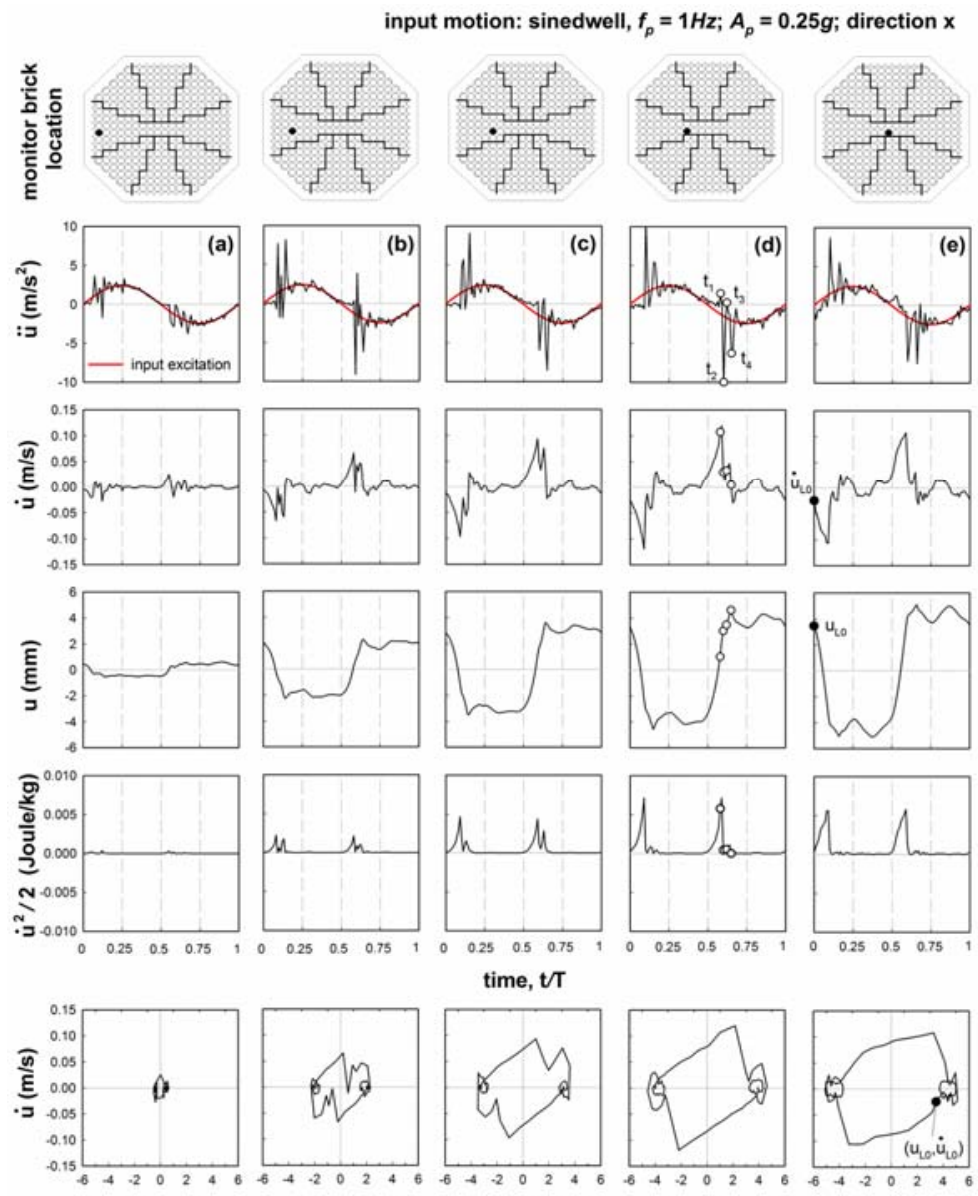

time, $\mathrm{tT}$
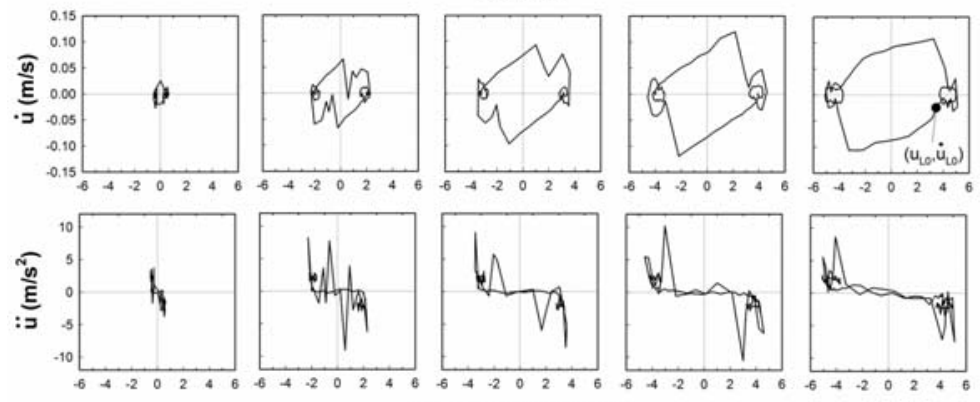

displacement, $\mathbf{u}(\mathrm{mm})$

Figure 12. Numerically evaluated lattice brick steady-state response time history segments along the $x$ axis

It is evident from the time histories in Figure 12 that the response is increasing towards the array centre ((a) to (e)), as would be expected. The displacements seem to be confined: they do not increase above a certain level and the limits are correlated with high frequency spikes on the acceleration response time histories, which are due to impacts/interactions between the bricks and the keying system. The same effect is apparent in the mass-normalised kinetic energy flow space $\left(\dot{u}^{2} / 2, t\right)$ demonstrated as significant kinetic energy loss in the system during impacts (sudden drops in the ordinates of the graphs) as well as from the hysteretic loops in the force-displacement space. The response exhibits some differences in amplitude per location, yet it is consistent along the $x$ symmetry axis. Also, the phase difference between excitation and response is more prominent towards the centre of the array. The numerical findings are again backed up by the experimental results, as depicted in Figure 13, where the predicted acceleration response of the LB nearest to the restraint 
frame is compared with the time history recorded by the closest restraint frame accelerometer. Note that the highfrequencies present in the recorded data are due to the sampling time step of $2 \times 10^{-4} s$ while only a $10^{-2} s$ time step is used for the numerical data. In addition, the frame accelerometer displays additional noise since it records interactions with all the bricks in the vicinity of the probe.

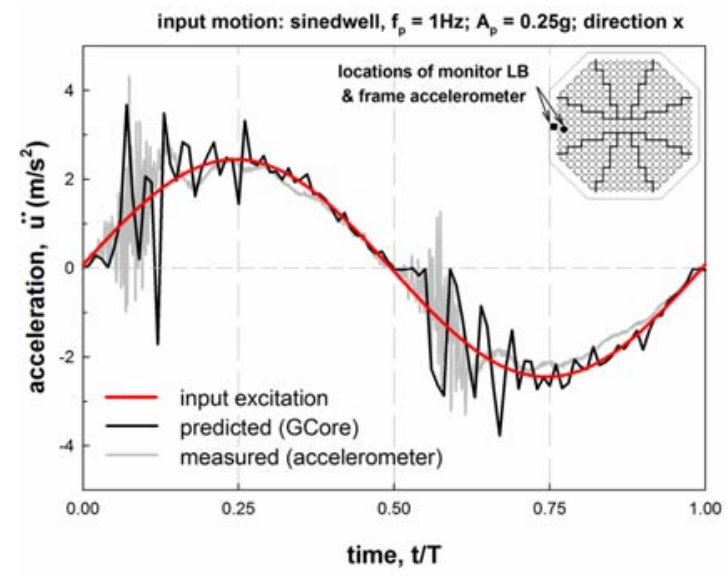

Figure 13. Comparison of predicted and recorded accelerations in the vicinity of the rigid frame.

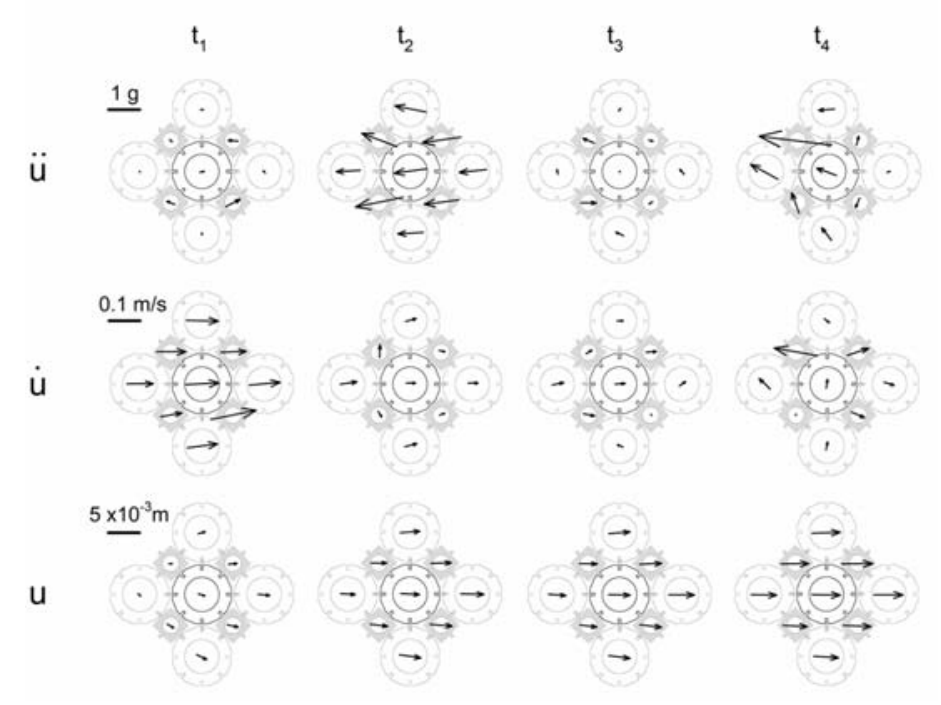

Figure 14. Acceleration, relative velocity and relative displacement vectors showing the interaction mechanisms between the central/monitored lattice brick to its adjacent lattice and interstitial bricks at specific time instants.

The lattice brick in Figure 12(d) was selected for a more comprehensive presentation. Its interactions with the neighbouring lattice and interstitial bricks at time instances $t_{1}-t_{4}$ in terms of acceleration, velocity and displacement vectors are plotted in Figure 14. Time instances $t_{1}-t_{4}$ correspond to characteristic brick acceleration peaks (Figure 12d). Specifically:

- $t_{l}$ is the time of maximum velocity during this excitation cycle. Note that all neighbouring bricks at $t_{l}$ have the same velocity orientation.

- $t_{2}$ is indicative of keying system interlocking to be referred to as "indirect impact" as there is no direct contact between adjacent lattice bricks. This is illustrated in Figure 14 as maximum acceleration values accompanied by kinetic energy loss, as brick velocities are significantly decreased. This is also evident by the sudden drop in the mass-normalised kinetic energy flow space $\left(\dot{u}^{2} / 2, t\right)$ in Figure 12.

- $t_{3}$ is a time instant after the impact when the keying system is still engaged. Figure 14 suggests that between $t_{2}-t_{3}$ all four lattice bricks of the system are moving uniformly (same velocities), which indicates that the keying system has been fully activated.

- $t_{4}$ is a second acceleration peak during which the keying system unlocks. The velocity vectors of the bricks on the left are reversed as shown in Figure 14. This seems to release the keying system allowing the interacting bricks to 
respond independently. $t_{4}$ also corresponds to the peak displacement response during this excitation cycle as the displacement between $t_{1}$ and $t_{4}$ is increasing monotonically and reaches a peak value at $t_{4}$.

The sensitivities of the numerical and physical models to the input motion characteristics (i.e. frequency and acceleration amplitude) is demonstrated through 3D visualisations (response maps) of the array response (i.e. acceleration, velocity, displacement). The following peak predicted steady-state response maps correspond to the top layer, as this is both the most mobile, exhibiting the maximum calculated and recorded displacements, and the most densely instrumented. A comparison of numerically and experimentally constructed maps is shown in Figure 15. Peak $x$ displacements, relative to the rigid base/frame, for input along the $x$-axis are presented at the four instrumented lattice column locations. Based on the shapes of the response maps, the physical and numerical models generally exhibit similar dependence on the input characteristics, although measured peak values are generally smaller than the predicted ones. The displacement plateau could be explained as an effect of "full" key engagement leading to horizontal lockup across the core, an effect that will be explained in detail in the following sections. Following the same logic, an explanation for the dip in displacement at $5 \mathrm{~Hz}$ and $0.05 \mathrm{~g}$ is that the brick displacements are too small to generate key engagement and therefore the columns rock essentially independently.
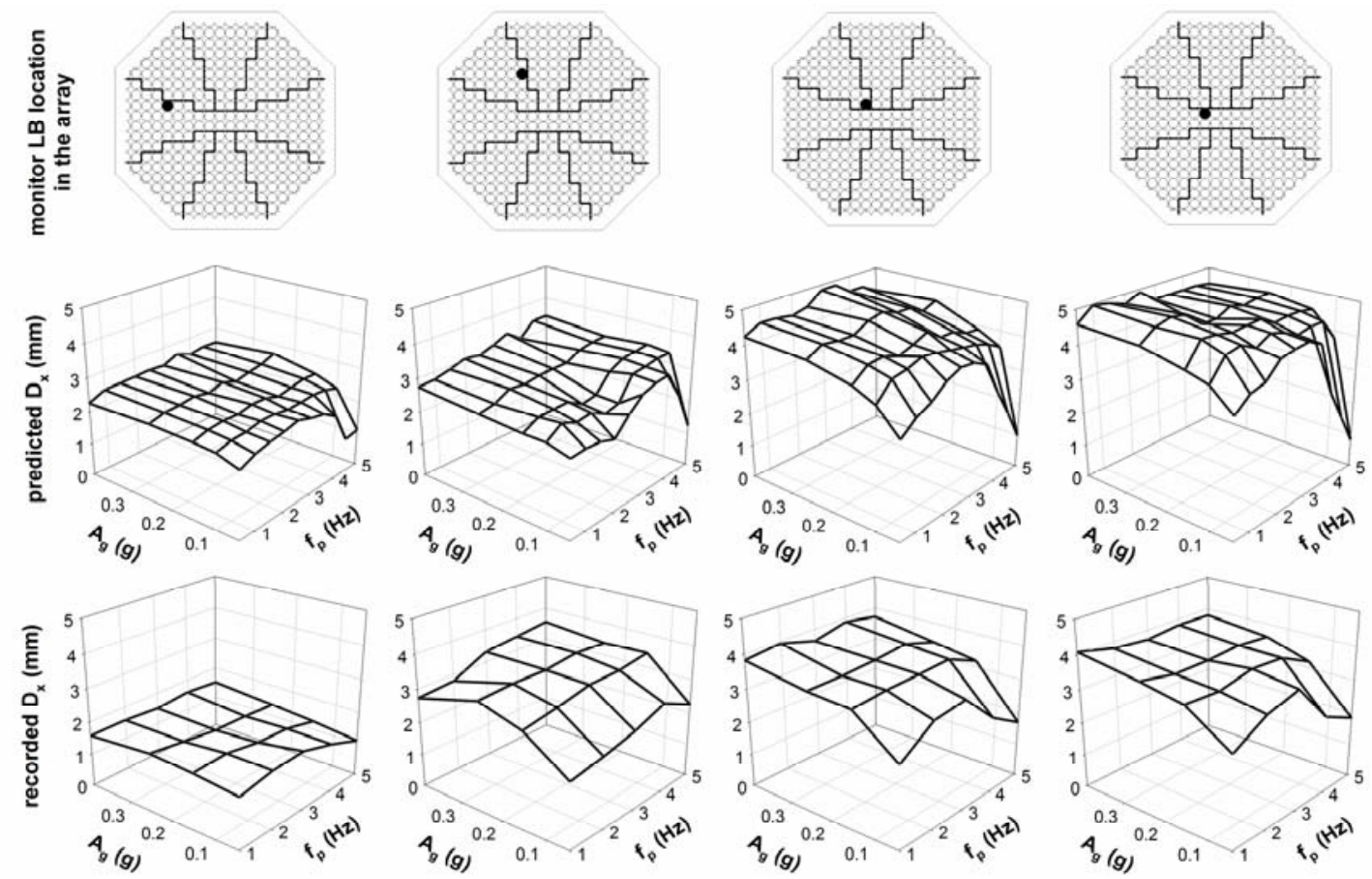

Figure 15. Comparison of numerical and experimental results: Peak relative displacements along $x$ directions of selected LBs at the top layer of the array.

To visualize the overall response of the top layer, seventeen locations were selected on the four bisectors of the rocking zones, distributed at three radii: adjacent to the frame, at the centre and at midrange as shown in Figure 16. On the bisectors which align with the $x$ and $y$ axes, the average response is reported for mirrored lattice bricks. The following observations can be made: (1) displacement response is symmetrically distributed; (2) peak displacement response is obtained at the centre, gradually decreasing towards the boundary following a radial distribution; (3) rocking orientation is affecting the response; (4) the effect of input acceleration and frequency is larger at the center; (5) resonance effects around 3-4Hz at model scale are evident for the lowest acceleration level of $0.05 g$; for larger input accelerations the displacement response seems to be largely independent of the frequency of the input motion.

Close to the centre of the array, where the maximum peak displacement values are encountered, the corresponding response maps for velocities and accelerations are plotted in Figure 17. The insensitivity of peak displacement on input frequency is not detected in the cases of peak velocity and acceleration. The kinematics of the array and the cut-off in displacement values dictated by geometry and the boundaries are discussed in detail later in this article. A useful remark drawn from Figure 17 is that although displacement is limited by geometry, the corresponding velocity and acceleration values are not likewise bounded. In other words, the displacement response is controlled by the boundary conditions adding to the stability of the array, but the energy transfer and the impacts between the array components increase with the input motion magnitude, thus affecting different aspects of its performance. 


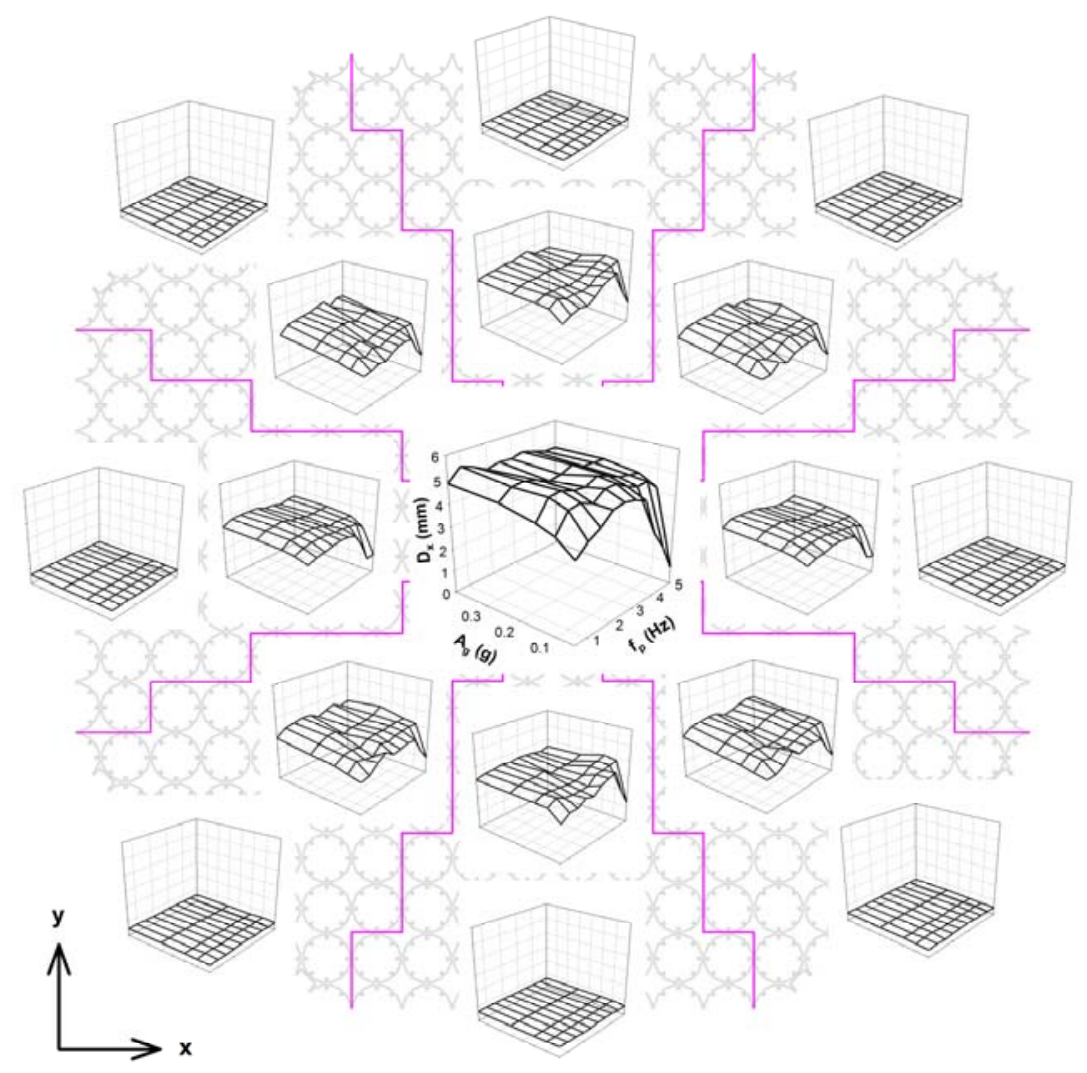

Figure 16. Predicted peak $x$ displacement response maps for harmonic excitation along the $x$-axis, at significant locations of the top layer.
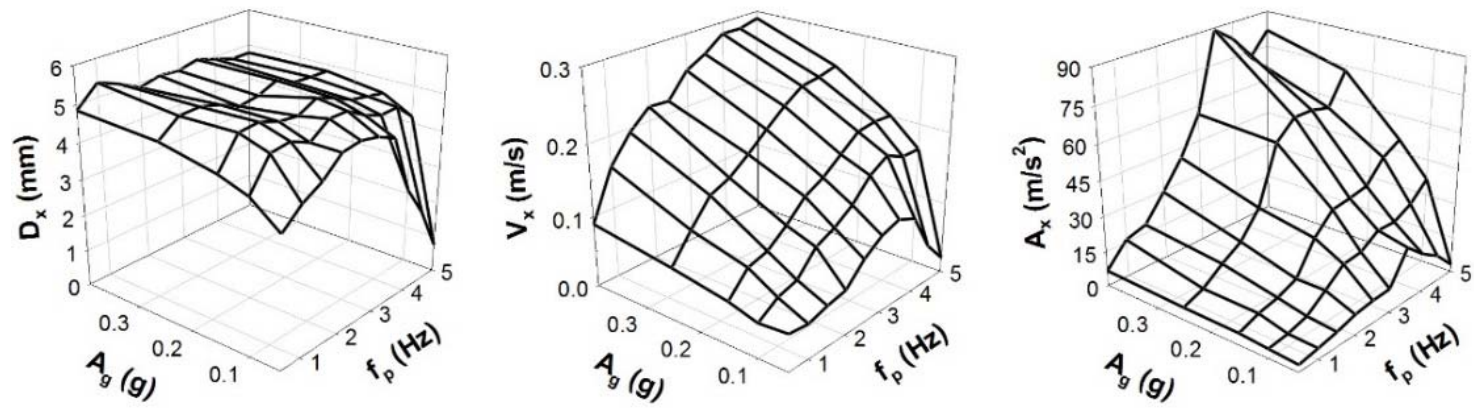

Figure 17. Predicted peak $x$ displacement, velocity and acceleration response maps for the centre of the array. Input motion on $x$ axis.

To obtain a better understanding of the mobility of the array, a visualization of the separations between the lattice bricks (brick-to-brick relative displacement) in all layers is provided in Figures 18-19. Three characteristic input excitations are considered, two harmonic and one seismic. For the harmonic input, the two cases having $A_{g}=0.25 g, f_{p}=1 H z$ and $A_{g}=0.05 g$, $f_{p}=1 \mathrm{~Hz}$ are presented as examples of strong and low amplitude excitations respectively. The seismic input is again the $H P B$ case specific earthquake. Figure 18 presents the envelope of maximum separations between adjacent lattice bricks along $x$ and $y$ axis over the duration of the input motion; the envelope defines the array zones of major brick interactions in each layer. The numerical model appears to respond in a symmetric manner with components close to the boundary generally exhibiting the largest separations. Towards the centre of the array, symmetry forces the bricks to move together, thus suppressing brick-to-brick relative movements. This pattern is consistent in all array layers for response along the $x$ axis in the case of the stronger harmonic input motion. For the cases of the HPB seismic input and the lower amplitude sinedwell, central bricks separate, especially at the lower layers of the array. A possible explanation is that the low excitation amplitude does not lead to extensive interlocking of the bricks, thus allowing different areas to move independently during shaking. For stronger excitations, the kinematic constraints of the model appear to control the response, accumulating the brick tolerances towards their limits. In the perpendicular $(y)$ direction, a gradual change in 
behavior is observed through the layers. At the bottom layer the $y$ separations clearly follow the rocking separation patterns, which is in agreement with the behaviour previously shown in Figure 13. The rocking effect disappears towards the top of the array, where the zones of maximum separations along $y$ coincide with those along $x$.

\section{Maximum LB-LB relative displacement $(\mathrm{mm})$}

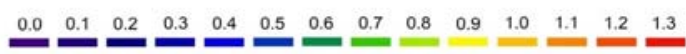

$\mathrm{X}$

L8

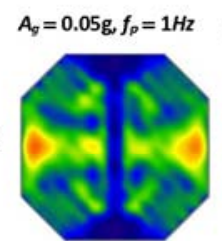

L7

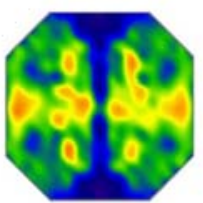

L6

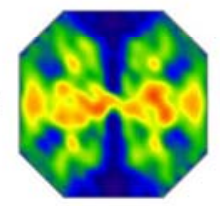

L5

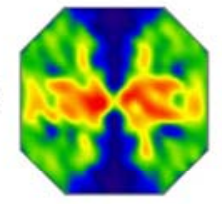

L4

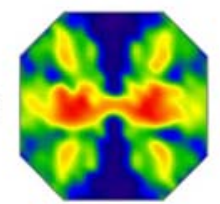

L3

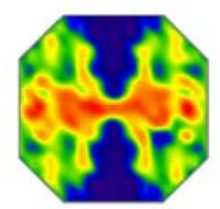

L2

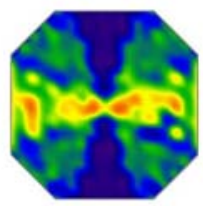

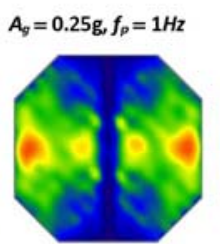
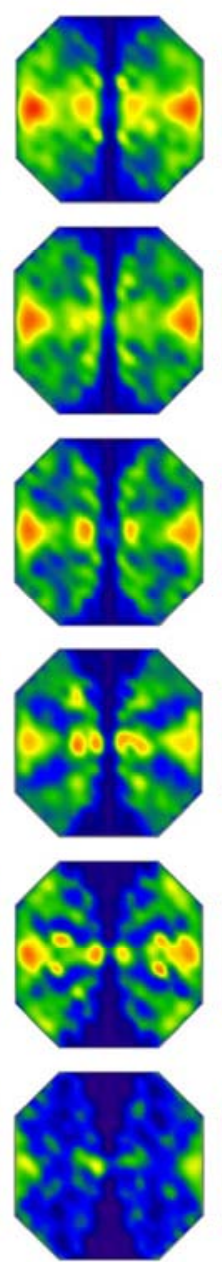
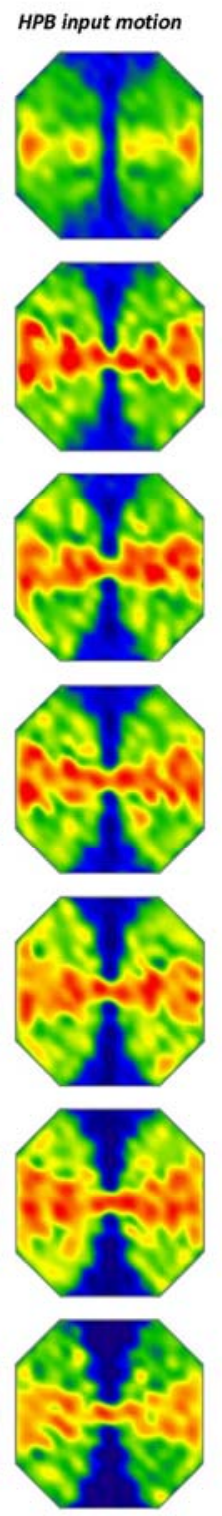
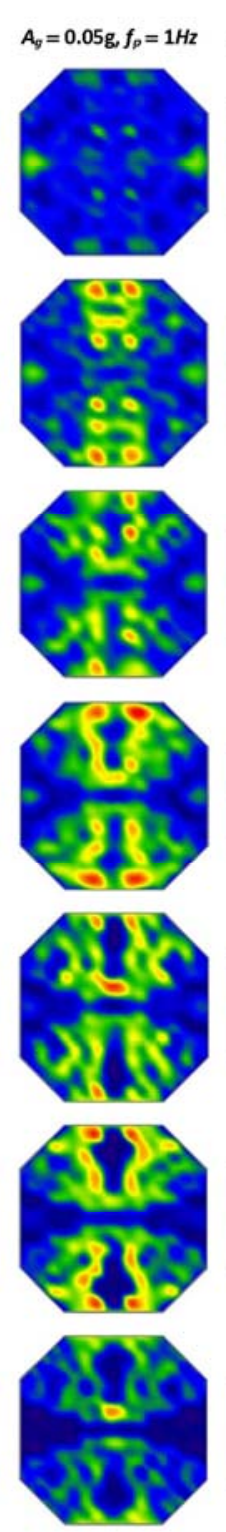

$Y$
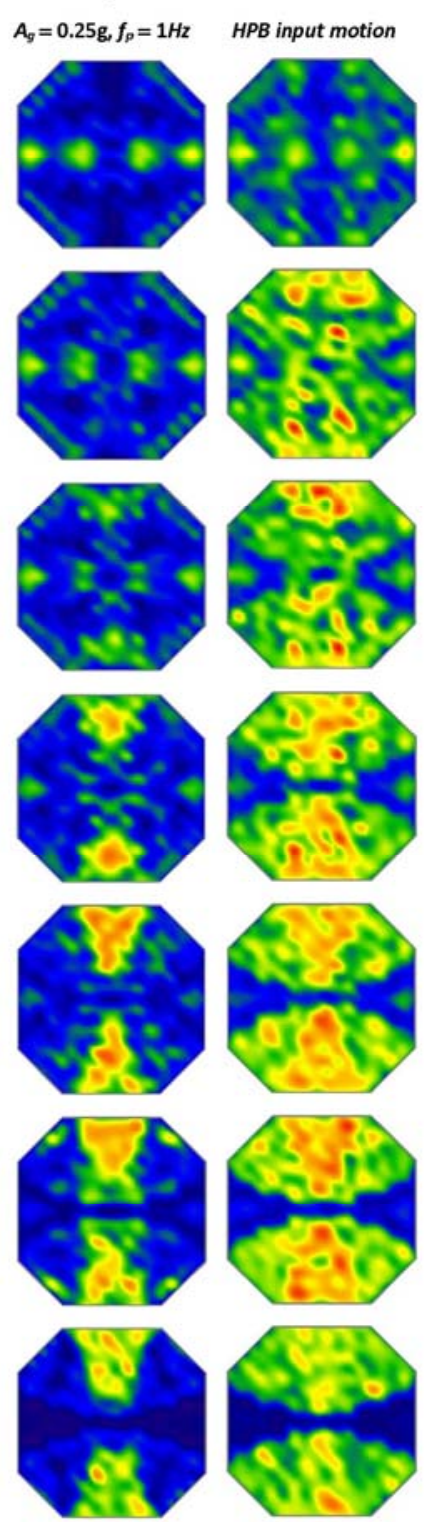

Figure 18. Visualisation of maximum brick-to-brick separation envelopes along $x$ and $y$ directions in layers 2-8 considering the HPB earthquake and two cases of harmonic input excitations along $x$.

Figure 19 presents the brick-to-brick separation profiles at the time of maximum relative-to-frame displacement at the center of the array's top layer. Symmetric expansion (+) and contraction (-) zones are created on the left and right parts of the array, respectively. Maximum separations at the extremity of the array (close to the frame) start to develop from the bottom layers 2 and 3. The bricks located close to the centre of the array gradually move apart and reach their limit separations towards the top layers. This reflects the tendency of the array to expand and contract also in the $y$ axis, to maximise movement in the $x$ axis. This pattern is reminiscent of the dilatancy effect in a densely packed granular medium, first proposed by Osborne Reynolds [47]. Separations in the $x$ and $y$ directions are thus kinematically coupled. From the $x$ response profiles, it is evident that the array responds in a "first mode" fashion, with $L B-L B$ separation values and distribution increasing towards the top of the array. This is an expected behaviour considering that the frequency content 
of the input motion is much smaller compared to the fundamental frequency of the model, to mobilise higher response modes. This behaviour is systematically observed in all herein studied cases.

Instantaneous LB-LB relative displacement ( $\mathrm{mm})$

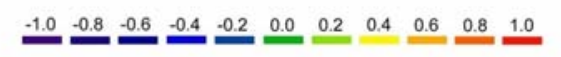

X

L8

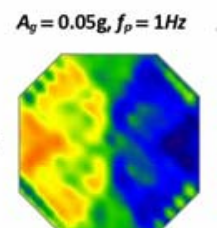

L7

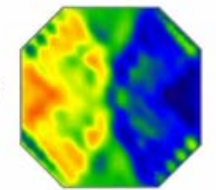

L6

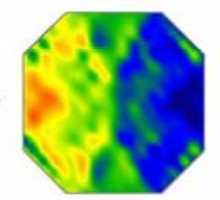

L5

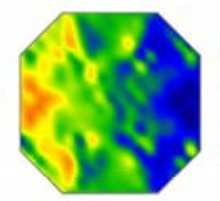

L4

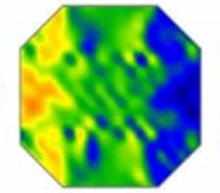

L3

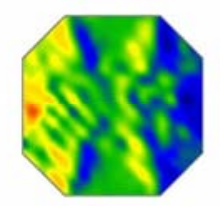

L2

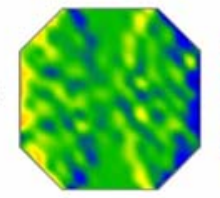

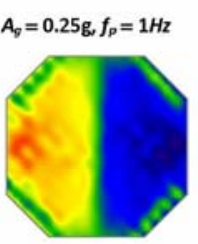
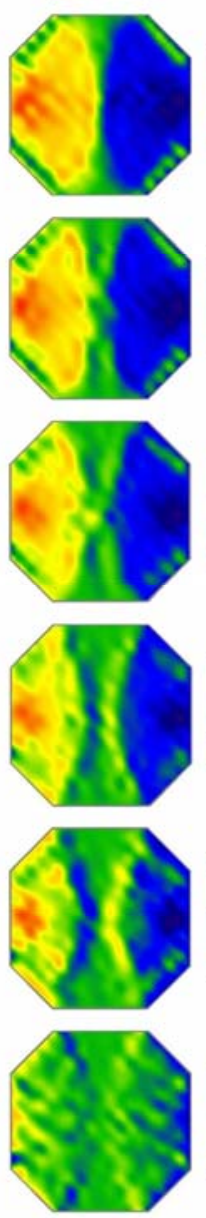

HPB input motion
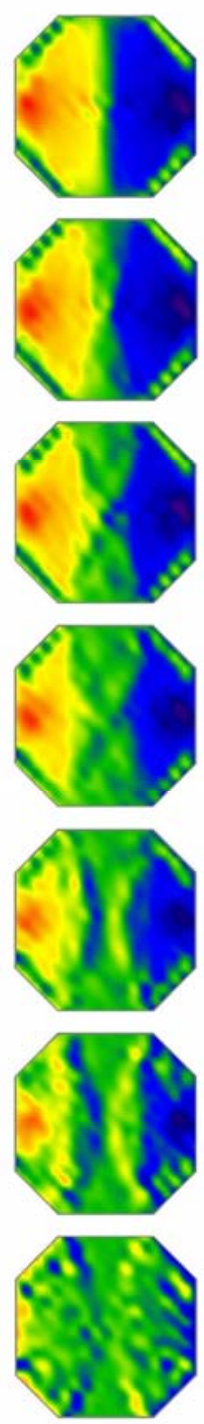
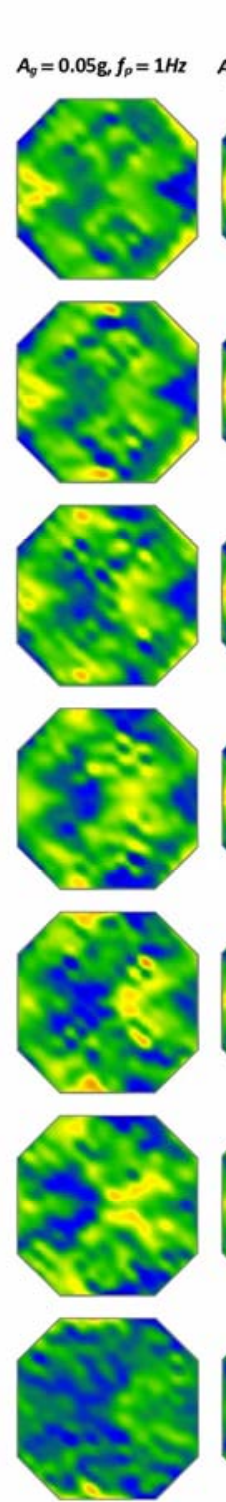

$Y$
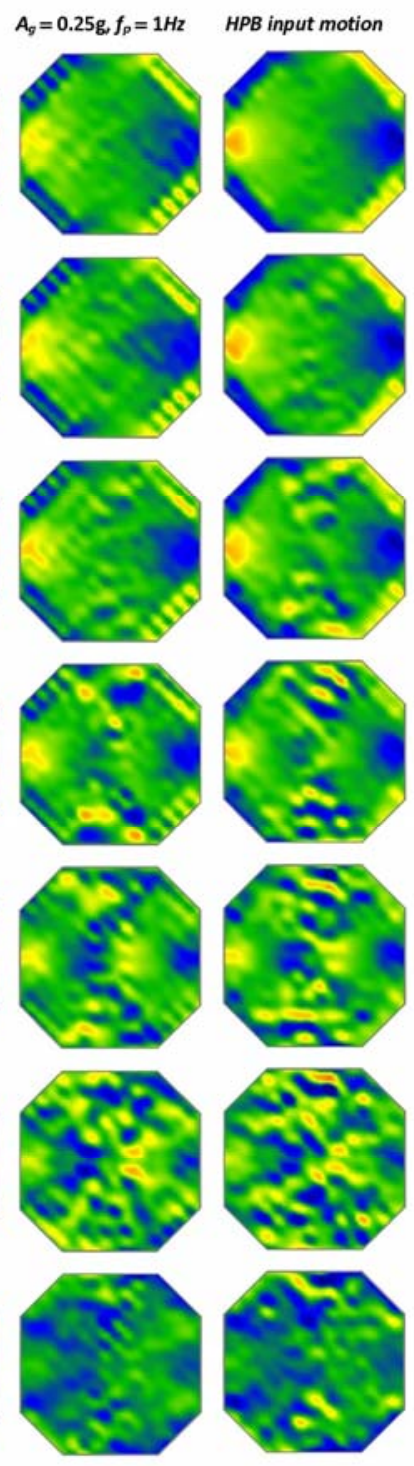

Figure 19. Visualisation of instantaneous brick-to-brick separation profiles, along $x$ and $y$ directions, at the time of maximum displacement relative to frame, at the center of layer 8 , considering the HPB earthquake and two cases of harmonic input excitations along $x$.

The accumulation of brick-to-brick separations to produce peak relative-to-frame LB displacement profiles at the centre of the top layer is shown in Figure 20 for the same input excitations as discussed above. Although maximum brick-tobrick separations (shown in Figs 18-19) occur at the outer edges of the array, peak column displacements are achieved in the centre of the array. The displacement is increasing to the maximum, not only from the perimeter to the center but also from the bottom to the top of the array, in a sense of a "first mode" response for the array columns. The relative displacement basic patterns for both the higher input acceleration harmonic input and the HPB input motion are remarkably similar, suggesting that the non-stationarity of the latter has no significant effect on the distribution of the array response. The coupling between $x$ and $y$ displacements is also evident from the distinct symmetric areas of out-ofplane motion activated along the $y$ direction. An interpretation of the foregoing in terms of the kinematics of the array is presented in the following section. 
Relative displacement, $D_{x}(\mathrm{~mm})$

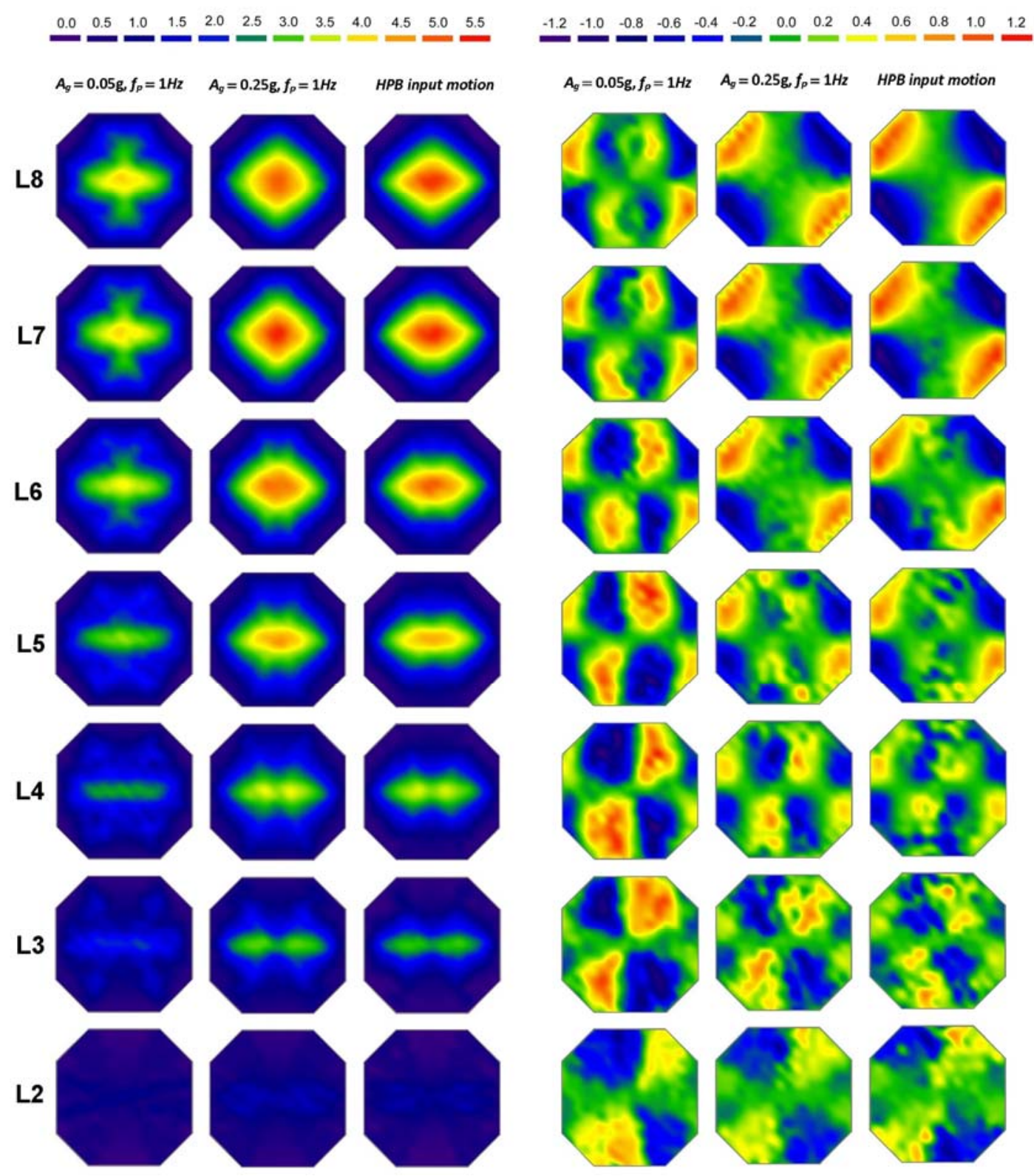

Figure 20. Visualisation of displacements relative to the rigid frame in layers 2-8, along $x$ and $y$, at the time instant of peak response at the center of layer 8, considering the HPB earthquake and two cases of harmonic input excitations along $x$.

\section{PROPOSED SIMPLIFIED 2-D KINEMATIC MODEL}

As shown in Figures 18-19, absolute peak calculated values of brick-to-brick separation/approaching are of the order of $1.3 \mathrm{~mm}$, which is significantly smaller than the $3.96 \mathrm{~mm}$ brick-to-brick direct contact gap of the model (Table 4). This suggests that direct contact between the LBs never occurs. Brick-to-brick interaction occurs only through the keying system. The shear deformations between the bricks are activating the integral (LB-IB interaction) and loose (LB-LB interaction) keys that, together with the rigid frame, control the overall stability of the array. A kinematic model for predicting the maximum potential deformations in the array's top layer, which is the most mobile, based on the shear clearances of the model and the numerical results, is therefore desirable. 

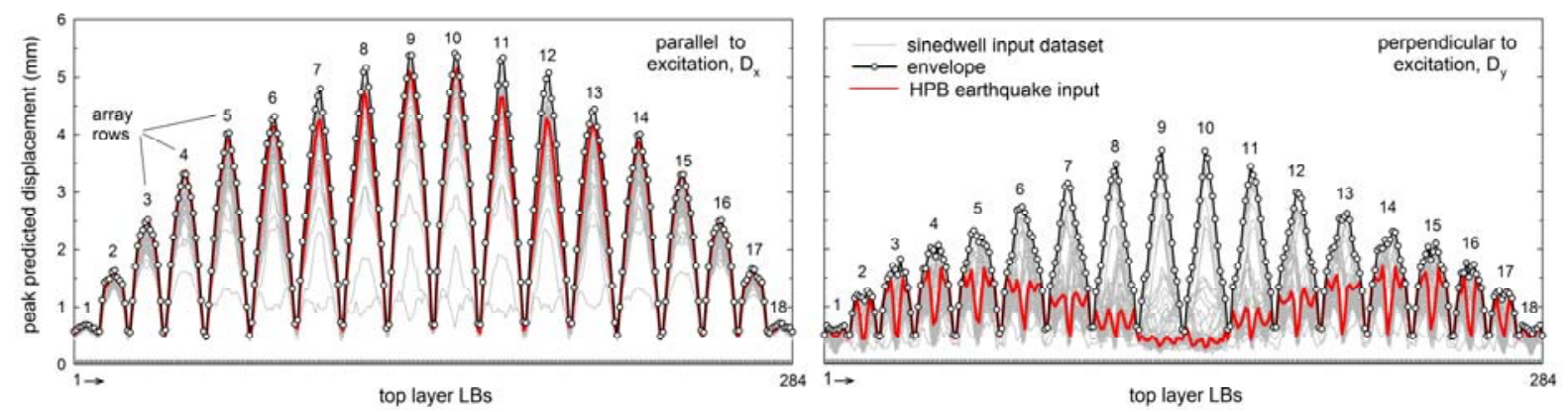

Figure 21. Peak LB displacements, relative to frame, during the 50 sinusoidal dwell inputs considered, exciting the system along the $x$ direction, each with a different acceleration amplitude. Grey lines indicate peaks during individual records. The black line is the envelope of all peaks. The red line indicates peaks during HPB earthquake motion.

Figure 21 depicts the numerically evaluated peak $D_{x}$ and $D_{y}$ displacements, relative to the restraint frame, for the whole 50 sinedwell dataset and the envelope of all calculated peaks. The peak displacements under the HPB earthquake motion, shown in red, are confined by the envelope of harmonics. The results are plotted for all 284 LBs in the arrays' top layer; numbers 1 to 18 indicate array rows, with 1 referring to the lowest $y$. The response is symmetric and increases both with the number of LBs per row and the distance from the restraint frame. For example, rows 5 to 14 have an equal number of $\mathrm{LBs}$, but the response is still increasing towards the centre of the array. This pattern is evident in both axes.

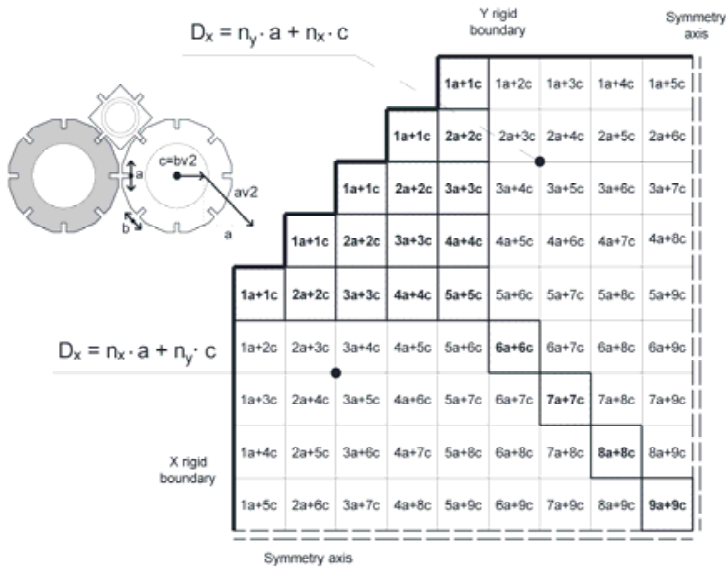

(a)

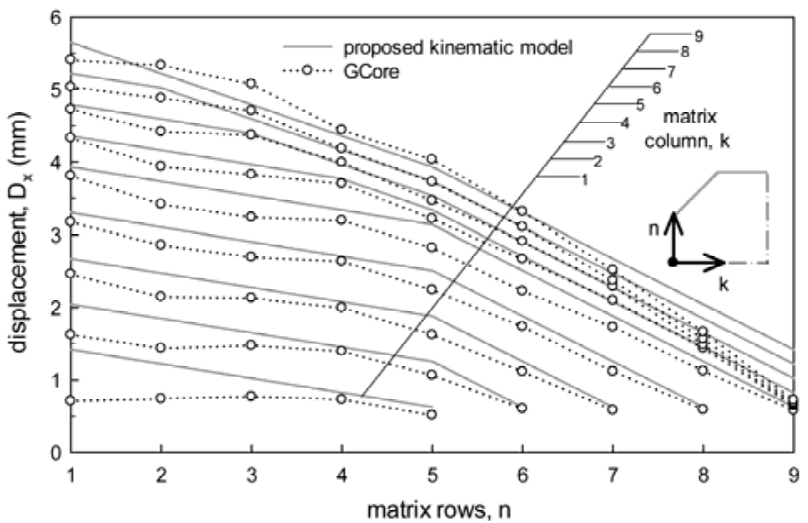

(b)

Figure 22. a) Proposed kinematic model matrix and b) comparison with numerically calculated displacement envelope.

An LB may move relative to its adjacent components following the simplified model illustrated in Figure 22, exhausting the shear clearances, referred to as "lost motion" values in Table 4. According to the proposed simplified kinematic model, the LB will initially move parallel to the $\mathrm{x}$ axis by $c=b \sqrt{2}$, exhausting the LB-IB interaction shear key/keyway backlash, which in GCORE equals $b=0.14 \mathrm{~mm}$. Then it will move parallel to the $45^{\circ}$ integral keying system until it exhausts the LBLB interaction shear key/keyway backlash, which in GCORE equals a $=0.43 \mathrm{~mm}$. Thus, its total horizontal movement potential is simply a $+c=0.63 \mathrm{~mm}$. Note that this is based on a purely translational kinematic mechanism ignoring any rotation induced displacements. To calculate the displacement potential of a given LB in the top layer of the array, one may merely aggregate the displacement potentials of all rows and columns between the brick under consideration and the rigid boundaries, according to the scheme shown in Figure 22. All other layers accumulate smaller displacements gradually decreasing down to the base. The proposed displacement function is summarised in Eq. (2)

$D_{x, p}\left(n_{x}, n_{y}\right)=\min \left(n_{x}, n_{y}\right) \cdot \mathrm{a}+\max \left(n_{x}, n_{y}\right) \cdot \mathrm{c}$

where $D_{x, p}\left(n_{x}, n_{y}\right)$ is the $x$ displacement potential of a LB located $n_{x}$ and $n_{y}$ LBs away from the X and Y rigid boundaries shown in Figure 22 respectively. Evidently, both boundaries govern the response, with LB-LB interactions being combined considering the relative shear deformation and the LB-IB interactions being combined considering the relative normal deformations. A comparison of the proposed kinematic model to the GCORE computed envelopes is shown in Figure 22(b). Predictions are generally good, but slightly overestimate the computed response in most cases, with the exception of the central array area where there is a slight under-prediction. A corresponding kinematic matrix for the peak $y$ response (perpendicular to the direction of excitation) can be produced, by combining the $y$-potential displacements also 
according to the basic kinematic mechanism shown in Figure 22a. For example, the maximum displacement at the center can be taken as 9 times the LB-LB shear keyway backlash to give value of $3.87 \mathrm{~mm}$, that matches the peak $D_{y}$ plotted in Figure $21 \mathrm{~b}$. Note that even though there are many possible ways these out-of-plane $y$ displacements aggregate from the boundary towards the center, their contribution on $D_{x}$ remains unaffected.

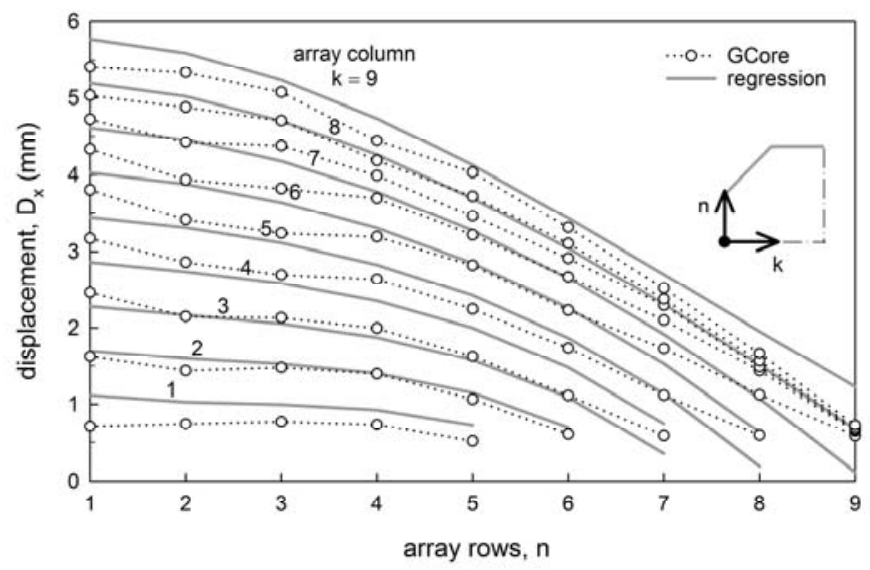

Figure 23. Comparison of the predicted displacement response envelopes by GCORE with the proposed equation calculated via regression of the numerical data.

An alternative way of deriving a predictor equation for the displacement potential of the core is through regression analysis of the GCORE results. The numerically produced envelope curves can be cast in the form of the third degree polynomial

$D_{x}=c_{0}+c_{1} n+c_{2} n^{2}+c_{3} n^{3} \quad($ in $m m)$

Eq. (3) was first fitted to produce discrete numerical values for the coefficients $c_{0}, c_{1}, c_{2}, c_{3}$ (in $m m$ ). These were then linearly fitted to produce Eqs. 4(a)-(d)

$c_{0}=0.8+0.55 k \quad, \quad c_{1}=-0.4+0.06 k \quad, \quad c_{2}=0.15-0.03 k \quad, \quad c_{3}=-0.018+0.0026 k$

In the above equations, $n$ and $k$ denote rows and columns in the array, respectively, measured from the symmetry axis. Comparisons between fitted and GCORE simulations results are provided in Figure 23. Evidently, the regression formula is a good predictor of peak displacement potential in the central area of the array. The above simple analyses illustrate how the interlocking geometry controls the gross behaviour of the array

\section{CONCLUSIONS}

An investigation of the dynamic response of a quarter scale nuclear core graphite array model to harmonic and synthetic earthquake input excitations has been presented. The problem is characterised by the controlled rocking response of multi - body column arrays connected by a shear inducing keying system. The experimental and numerical data are used to understand the salient features of the dynamics of the multi block core. The main conclusions of the study are summarised in the following:

1) Displacement response is symmetrically distributed. Peak displacements are obtained at the central areas of the core's top layer, gradually decreasing towards the boundaries following a radial distribution.

2) Although peak displacements relative to the boundaries are naturally recorded in the central zone, the larger brick-tobrick separations occur at the outer rings of the array close to the rigid boundary.

3) Rocking orientation is controlling the response mostly in the lower layers; towards the top layer the response aligns with the direction of excitation.

4) Regarding peak response parallel to the direction of excitation, resonance effects are evident between 3-4Hz at low input acceleration levels; for larger input accelerations displacements seem to be independent of excitation frequency. This is in contrast with the behaviour of classical non-linear yielding systems [11,47].

5) The characteristics of the harmonic input motion seem to affect the response for input acceleration values below $0.15 \mathrm{~g}$. In this case the response is governed by the dynamic properties of the system. For stronger excitations the kinematics of the model appear to control the response, by accumulating the brick separations towards the geometric limits.

6) Although displacements are limited by geometry, increasing the acceleration levels brings about higher velocities and forces, mostly due to impacts within the array.

7) The pounding of the core components to the rigid lateral boundary, which follows the movement of the base of the core, makes the relative-to-base accelerations (in addition to the relative velocities and displacements) a relevant parameter in this problem. 
8) Absolute peak calculated values of brick-to-brick separation/approaching are on the order of $1.3 \mathrm{~mm}$, which is significantly smaller than the $3.96 \mathrm{~mm}$ brick-to-brick direct contact gap of the model. This suggests that direct contact between the LBs never occurs in the presented case of the intact array. Brick-to-brick interaction is implicit through the keying system.

9) The interlocking of the array's keying system resembles that of a densely compacted granular matter which expands under combined compression and shearing. This coupling between shear and volumetric deformations, known as Reynolds dilatancy [48], is not typical in structural dynamics yet is evident in the response of the particular system.

10)Based on this dilative behaviour, a simplified kinematic model was proposed for estimating the peak displacement potential of the core. Predictions of the kinematic model are generally good, slightly exceeding the numerical calculations in most cases. A more accurate predictor of the response potential of the central array zones was derived by means of a polynomial regression formula of the numerical data. Comparisons with both methods are satisfactory.

11)The numerical findings are confirmed by comparisons with experimental data. The intrinsic response mechanisms predicted by GCORE are also present in the physical model. As not all keys, interactions and damping mechanisms are implemented in the numerical model, GCORE consistently, yet slightly, over-predicts the mobility of the array.

12) Despite the complexity of the 35,000 non-linear dof model, including the large number of interactions between the array components $\left(>10^{3}\right)$, the non-linearity of contacts, the number of degrees of freedom, and the variety of input excitations considered, the array response is evidently symmetric, repeatable and consistent. This is in contrast to the highly complex/non-reproduceable response of a small number of rocking blocks $\left(<10^{1}\right)$, such as multi-drum classical columns, and anchored electrical equipment, the behavior of which is often referred to as "chaotic" [49]. The repeatable/predictable response can be interpreted as a non-linear "smoothening" process due to the large number of associated dofs. This is in accordance with the consistency and repeatability of dynamic geotechnical laboratory tests, such as cyclic triaxial and simple shear, despite the complexity of interparticle interaction mechanisms at micro scale.

13)Following up on the above point, the observations reported in this study raise the fundamental question as to what is the critical number of dynamic rocking components that maximises complexity in the response. To the best of the authors knowledge this has not been addressed in the literature.

The findings of this study provide important support to the validation of the GCORE modelling approach when applied the behaviour of actual AGR graphite cores. A follow-up paper will address additional issues including the behaviour of a cracked array due to radiation and temperature effects.

\section{ACKNOWLEDGMENTS}

The authors acknowledge the financial support by EDF Energy and the permission to publish this paper. Thanks are given to Miss Sarah Corrigan and Miss Heather Riley of Atkins for providing technical support with GCORE and to Professor George Mylonakis for proofreading the manuscript. Special thanks are also due to Dr Elias Dimitrakopoulos for bringing to the attention of the authors the question of non-linear smoothening. The management of the project on behalf of UoB by Mrs Viv Paulete, the technical laboratory support of Mr Dave Ward, and the help of Dr Callum Wright in running BlueCrystal at University of Bristol Advanced Computing Research Center, are gratefully acknowledged.

\section{REFERENCES}

1) Housner GW. The behavior of inverted pendulum structures during earthquakes. BSSA 1963; 52(2): 404-17.

2) Yim CS, Chopra AK, Penzien J. Rocking response of rigid bodies to earthquakes. EESD 1980; 8: 565-587.

3) Ishiyama Y. Motion of rigid bodies and criteria for overturning by earthquake excitations. EESD 1982; 10: 635-650.

4) Psycharis IN, Jennings PC. Rocking of slender bodies allowed to uplift. EESD 1983; 11: 57-76.

5) Spanos PD, Koh AS. Rocking of rigid bodies due to harmonic shaking. J. Eng Mechanics, ASCE 1984; 110 (11): $1627-1642$.

6) Zhang J, Makris N. Rocking response of a free-standing blocks under cycloidal pulses. J. Eng Mechanics 2001; 127 (5): $473-483$

7) Prieto F, Lourenço PB, Oliveira CS. Impulsive Dirac-delta forces in the rocking motion. EESD 2004; 33 (7): 839-857.

8) Dimitrakopoulos E, DeJong M. Revisiting the rocking block: closed-form solutions and similarity laws. Proceedings of the Royal Society 2012; 468 (2144): 2294-2318.

9) Dimitrakopoulos E, DeJong M. Overturning of retrofitted rocking structures under pulse-type excitations. Journal of Engineering Mechanics, ASCE 2012; 138(8): 963-972.

10) Psycharis IN, Fragiadakis M, Stefanou I. Seismic reliability assessment of classical columns subjected to near-fault ground motions. Earthquake Engineering \& Structural Dynamics 2013; 42 (14): 2061-2079.

11) Voyagaki E. Contributions to dynamic analysis of yielding systems to near-fault earthquake motions, PhD Thesis, National Technical University, Athens, Greece, 2013

12) Voyagaki E, Psycharis IN, Mylonakis G. Rocking Response and Overturning Criteria for Free Standing Rigid Blocks to Single - Lobe Pulses. Soil Dynamics \& Earthquake Engineering 2013; 46: 85-95.

13) Voyagaki E, Psycharis IN, Mylonakis G. Complex Response of a Rocking Block to a Full-Cycle Pulse. Journal of Engineering Mechanics ASCE 2014; 140(16)

14) Voyagaki E, Vamvatsikos D. Probabilistic Assessment of Rocking Response for Simply-Supported Rigid Blocks. SECED 2015 Conference: Earthquake Risk and Engineering towards a Resilient World, Cambridge, UK, 1-9 July, 2014.

15) Vassiliou MF, Burger S, Egger M, Bachmann JA, Broccardo M, Stojadinovic B, The three-dimensional behavior of inverted pendulum cylindrical structures during earthquakes. Earthquake Engineering \& Structural Dynamics 2017; 46(14): 2261-2280.

16) Muto K, Umemura H, Sonobe Y. Study of overturning vibration of slender structures. 2WCEE, Tokyo, Japan, 1960. 
17) Aslam M, Godden WG, Scalise DT. Earthquake rocking response of rigid bodies. Journal of the Structural Division, ASCE 1980; 106 (ST2): 377-392.

18) Tso WK, Wong CM. Steady state rocking response of rigid block. Part II: Experiments. EESD 1989; 18, 107-20.

19) Anooshehpoor A, Brune JN, Zeng Y. Methodology for Obtaining Constraints on Ground Motion from Precariously Balanced Rocks. BSSA 2004; 94 (1): 285-303.

20) Peña F, Prieto F, Lourenço PB, Campos Costa A, Lemos JV. On the dynamics of rocking motion of single rigid-block structures. EESD 2007; 36: 2383-2399.

21) Allen RH, Oppenheim IJ, Parker AP, Bielak J. On the dynamic response of rigid body assemblies. Earthquake Engineering \& Structural Dynamics 1986; 14, 861-876.

22) Sinopoli A. Dynamic evolution by earthquake excitation of multiblock structures. Proc. Int.Conference on structural conservation of stone masonry: diagnosis, repair \&strengthening. Greek Ministry of Culture, Athens, 1989.

23) Psycharis IN. Dynamic behaviour of rocking two-block assemblies. EESD 1990; 19: 555-575.

24) Winkler T, Meguro K, Yamazaki F. Response of rigid body assemblies to dynamic excitation, EESD 1995; 24: 1389-1408.

25) Psycharis IN, Papastamatiou DY, Alexandris A. Parametric investigation of the stability of classical columns under harmonic and earthquake excitations. Earthquake Engineering and Structural Dynamics 2000; 29: 1093-1109.

26) Spanos PD, Roussis PC, Politis NPA. Dynamic analysis of stacked rigid blocks. SDEE 2001; 21(7): 559-78.

27) Mouzakis H, Psycharis IN, Papastamatiou DY, Carydis PG, Papantonopulos C, Zambas C. Experimental investigation of the earthquake response of a model of a marble classical column. EESD 2002; 31: 1681-1698.

28) Papantonopoulos C, Psycharis IN, Papastamatiou DY, Lemos JV, Mouzakis H. Numerical prediction of the earthquake response of classical columns using the Distinct Element Method. EESD 2002; 31: 1699-1717.

29) Psycharis IN, Lemos JV, Papastamatiou DY, Zambas C, Papantonopoulos C. Numerical study of the seismic behaviour of a part of the Parthenon Pronaos. EESD 2003; 32: 2063-2084.

30) Konstantinidis D, Makris N. Seismic response analysis of multidrum classical columns. EESD 2005;34: 1243-1270.

31) Dasiou ME, Mouzakis HP, Psycharis IN, Papantonopoulos C, Vayas I. Experimental investigation of the seismic response of parts of ancient temples. Prohitech conference, Rome, 21-24 June 2009.

32) Papaloizou L, Komodromos P. Planar investigation of the seismic response of ancient columns and colonnades with epistyles using a custom-made software. Soil Dynamics and Earthquake Engineering 2009; 29: 1437-1454.

33) Ambraseys N, Psycharis IN. Earthquake Stability of Columns and Statues. J. Earthquake Engineering 2011; 15(5): 685-710.

34) Ambraseys N, Psycharis IN. Assessment of the long-term seismicity of Athens from two classical columns, Bulletin of Earthquake Engineering 2012; 10: 1635-1666.

35) Muto K, Uchida K, Takase H, Kasai Y, Kuroda T, Murakami H. Two-dimensional vibration test and simulation analysis for a vertical slice model of HTGR core, $4^{\text {th }}$ SMIRT Conference, San Francisco, USA, Paper K7/8, 1977.

36) Ikushima T, Nakazawa T. A seismic analysis method for a block column gas-cooled reactor core, NED 1979; 55: 331-342.

37) Ahmed KM. The Dynamic Response of Multi-Layer AGR Core Brick Arrays, Nuclear Engineering Design 1987; $104: 1-66$.

38) Ahmed KM, Parker JV, Proffitt DE. Seismic Response of the Advanced Gas Cooled Reactor Core, NED 1986; 94: 67-92.

39) Ahmed KM, Stojko S. The non-linear seismic response of AGR core graphite brick slices - correlation of experimental and analytical results, Earthquake Engineering and Structural Dynamics 1987; 15: 159-188.

40) Livermore Software Technology Corporation (LSTC). LS-DYNA software, http://www.lstc.com/products/ls-dyna, 2011.

41) Kralj B, Humphreys SJ, Duncan BGJ. Seismic Modelling of an AGR Nuclear Reactor Core, Proceedings of the Ageing Management of Graphite Reactor Cores, pp 193-200, Cardiff, UK, 28-30 Nov 2005.

42) Steer AG. AGR Core Design, Operation and Safety Functions, Proceedings of the Ageing Management of Graphite Reactor Cores, pp 11-18, University of Cardiff, UK, 28-30 Nov 2005.

43) Dihoru L, Oddbjornsson O, Horseman T, Dietz M, Wilson J, Kloukinas P, Voyagaki E, Crewe AJ, Taylor CA. Multi-Layer Array Rig Work for Seismic Behaviour With Cracked Bricks, The 4th EDF Energy Nuclear Graphite Symposium. Engineering Challenges Associated with the Life of Graphite Reactor Cores, Nottingham, UK, 2014.

44) Dihoru L, Oddbjornsson O, Kloukinas P, Dietz M, Horseman T, Voyagaki E, Crewe A, Taylor C, Steer A. The Development of a Physical Model of an Advanced Gas Cooled Reactor Core: Outline of the Feasibility Study. Nuclear Engineering and Design 2017; 323: 269-279.

45) Oddbjornsson O, Kloukinas P, Dihoru L, Dietz M, Horseman T, Voyagaki E, AJ Crewe AJ, Taylor CA, Steer AG. Physical Modelling and Testing of an Advanced Gas Cooled Reactor Core Model, Paper No. 795 in Proceedings 16th World Conference on Earthquake Engineering, Santiago, Chile, 9-13 January 2017.

46) Principia Mechanica Limited. Seismic ground motions for UK design. Report for CEGB and BNFL. 1981.

47) Mylonakis G, Voyagaki, E. Yielding Oscillator subjected to simple pulse waveforms: numerical analysis \& closed - form solutions. Earthquake Engineering \& Structural Dynamics 2006; 35(15): 1949-1974

48) Reynolds O. LVII. On the dilatancy of media composed of rigid particles in contact, with experimental illustrations. Philosophical Magazine Series 5 1885; 20 (127): 469-481.

49) Bachmann JA, Strand M, Vassiliou MF, Broccardo M, Stojadinović B. Is rocking motion predictable? Earthquake Engineering \& Structural Dynamics 2017; 47(2): 535-552. 